\title{
Quantum liquid-crystal order in resonant atomic gases
}

\author{
Leo Radzihovsky \\ Department of Physics, University of Colorado, Boulder, CO 80309
}

(Dated: July 31, 2021)

\begin{abstract}
I review recent studies that predict a realization of quantum liquid-crystalline orders in resonant atomic gases. As examples of such putative systems I will discuss an s-wave resonant imbalanced Fermi gas and a p-wave resonant Bose gas. In the former, the liquid-crystalline smectic, nematic and rich variety of other descendant states emerge from strongly quantum- and thermally- fluctuating Fulde-Ferrell and Larkin-Ovchinnikov states, driven by a competition between resonant pairing and Fermi-surface mismatch. In the latter, at intermediate detuning the p-wave resonant interaction generically drives Bose-condensation at a finite momentum, set by a competition between atomic kinetic energy and atom-molecule hybridization. Because of the underlying rotationally-invariant environment of the atomic gas trapped isotropically, the putative striped superfluid is a realization of a quantum superfluid smectic, that can melt into a variety of interesting phases, such as a quantum nematic. I will discuss the corresponding rich phase diagrams and transitions, as well the low-energy properties of the phases and fractional topological defects generic to striped superfluids and their fluctuation-driven descendants.
\end{abstract}

\section{INTRODUCTION}

\section{A. Resonant atomic gases}

Experimental progress in trapping, cooling and coherently manipulating Feshbach-resonant atomic gases opened unprecedented opportunities to study degenerate strongly interacting quantum many-body systems in a broad range of previously unexplored regimes [1 5]. These include paired fermionic superfluids (SF) 6 15]), the associated Bardeen-Cooper-Schrieffer (BCS) to BoseEinstein condensation (BEC) crossover 3, 16 24, BoseFermi mixtures 25], bosonic molecular superfluids [26 29], and many other states and regimes 30 under both equilibrium and nonequilibrium conditions [23, 31, 32.

Because degenerate atomic gases are free of the underlying crystalline matrix of the solid-state materials (though one can be imposed through a highly tunable optical lattice potential[1]), among this rich variety of states, they admit phases that spontaneously partially break continuous spatial symmetries and thereby exhibit concomitant strongly fluctuating Goldstone modes with corresponding rich phenomenology. Resonant atomic gases are thus uniquely suited for a realization of quantum liquid-crystalline states of matter, that have been somewhat of a holy-grail dating back to their studies in solid state materials such as the striped states in high- $\mathrm{T}_{c}$ superconductors, nickelates and other strongly correlated doped Mott insulators 33 38, heavy-fermion and organic superconductors [39, 40, spiral states in helimagnets 4143 , and a two-dimensional electron gas with a partiallyfilled Landau level 44, 46, 47]. In isotropic traps the putative quantum liquid-crystal order is expected to exhibit all the complexity of fluctuations and topological defects of conventional (mesogenic) liquid crystals 48, 49, but with the added enrichment of the accompanying quantum (off-diagonal) order of a superfluid. Another, not insignificant virtue is that (in contrast to other e.g., solidstate or nuclear matter systems) these dilute gases are extremely well-characterized at the two-body level, and are therefore described by microscopic (as opposed to effective) Hamiltonians with well-known couplings.

\section{B. Candidate systems}

Recent theoretical studies have predicted a number of such quantum liquid crystal realizations in degenerate atomic systems, that in addition to internal symmetries partially break spatial symmetries [50 56]. These are typically driven by strong resonant and competing interaction that frustrates a spatially homogeneous and isotropic superfluidity. Known examples include bosonic and paired fermionic superfluids, where spatial order is driven by (i) dipolar interaction 60, (ii) pseudo-spinorbit interaction [57] (realized through hyperfine states coupled by Raman transitions [58]), (iii) p-wave resonant interaction 53, 59, and (iv) a Fermi surface mismatch (realized through species number and/or mass imbalance), that leads to the Larkin-Ovchinnikov-Fulde-Ferrell finite-momentum pairing [50, 51, as well as their strongly fluctuating descendent states. In this brief review, I will focus on the last two realizations, and will discuss the associated microscopic models that I believe can realize a quantum superfluid liquid-crystal order, their phase behavior, fluctuations, topological defects, and a variety of experimental predictions and signatures. Much of the discussed low-energy phenomenology is shared more generally by systems exhibiting quantum liquid crystal orders in isotropic trap [54]. For a more complete account, I refer the reader to the original literature and the more extensive reviews 2, 5.

I will not discuss the finite momentum states that depend on the lattice for their realization and stability, such as the p-band and FFLO superfluids in optical lattices 61 65. These are fascinating states, but are less relevant from the liquid-crystal perspective of this review. 


\section{IMBALANCED RESONANT FERMI GASES}

\section{A. Background}

The most widely explored candidate for a realization of quantum liquid crystal order is a species-imbalanced Feshbach-resonant Fermi gas, 2, 3, 5, though it is only very recently that it was formulated and explored in these liquid-crystal terms 66, 67. These studies build on well-explored two-species Feshbach-resonant Fermi gas, that exhibits paired superfluidity, that can be tuned between a weakly-attractive Fermi-surface-driven BCS and a strongly-attractive molecular BEC superfluids [3, 4]. While at $T=0$ a balanced gas exhibits no qualitative change of state, a quantitatively accurate description of this crossover, particularly around the strongly interacting and universal unitary regime (where in a vacuum a two-particle bound state first forms and the s-wave scattering length diverges) has presented a considerable challenge with much recent progress.

A species-number (and mass) imbalance in the two atomic hyperfine-states mixture offered a new extremely fruitful experimental knob 68 71]. The imbalance frustrates pairing [72 77, driving quantum phase transitions out of the paired superfluid to a variety of possible ground states and thermodynamic phases [52, 78,83$]$. This rekindled considerable theoretical activity in the context of species-imbalanced resonant Fermi gases 84 -106]. The corresponding imbalance versus detuning BCS-BEC phase diagram, illustrated in Fig.1 (and its extension to finite temperature) is now well-established $79,100,103$, showing qualitative agreement with experiments 68 71 . More recently, considerable progress has been made toward establishing quantitative details of this phase diagram through analytical[107-111], numerical[112, 113 . and experimental approaches 70, 71, 114.

The identification of the number species imbalance with the magnetization of an electronic system, and the chemical potential difference with an effective Zeeman energy, connects these atomic gases studies with a large body of research on solid state electronic superconductors under a Zeeman field [50, 51, 115, 117, as well as extensively studied realizations in nuclear and particle physics 118 121]. The obvious advantage of the newlyrealized atomic system is the aforementioned tunability, disorder-free "samples", and absence of the orbital part of the magnetic field, that always accompanies a solid-state charged superconductor in a magnetic field. In these neutral paired superfluids the orbital field effects can be independently controlled by a rotation of the atomic cloud[122].

As illustrated in Fig, 1, among many interesting features, such as the gapless imbalanced superfluid $\left(S F_{M}\right)$ 79 81, 101, ubiquitous phase separation 74, 79, 100, 101, tricritical point [100, 103, [106], etc., observed experimentally 68, 71] and studied extensively theoretically [5], the interaction-imbalance BEC-BCS phase diagram is also predicted 79, 81, 84, 100, 104,
105. to exhibit the enigmatic Fulde-Ferrell-LarkinOvchinnikov state (FFLO) [50, 51]. First predicted in the context of solid-state superconductors over 45 years ago, the FFLO states has so far eluded a definitive observation, though some promising solid state $[123$ and quasi- $1 \mathrm{~d}$ atomic 124] candidate systems have recently been realized.

At its most generic level the FFLO state is a fermionic superfluid, paired at a finite center of mass momentum. It spontaneously "breaks" gauge and translational symmetry, a periodically-paired superfluid (superconductor), akin to a supersolid [125]128, and thus can appropriately be called a pair-density wave (PDW) 129, 130. This state can be equivalently thought of as a periodically ordered micro-phase separation between the normal and paired states, that naturally replaces the macro-phase separation [74, 121] ubiquitously found in the BCS-BEC detuning-imbalance phase diagram [79, 100, 101, 103.

Microscopically, it is driven by Fermi surface mismatch [50, 51] due to an imposed pairing species number (and/or mass 132]) imbalance. As a compromise between the superfluid pairing and an imposed imbalance, at intermediate values of the latter, the superconducting order parameter condenses at a set of finite center-ofmass momenta determined by the details of the Fermi surface mismatch and interactions. At sufficiently large imbalance, no compromise is possible, and the resonant gas transitions to the normal state.

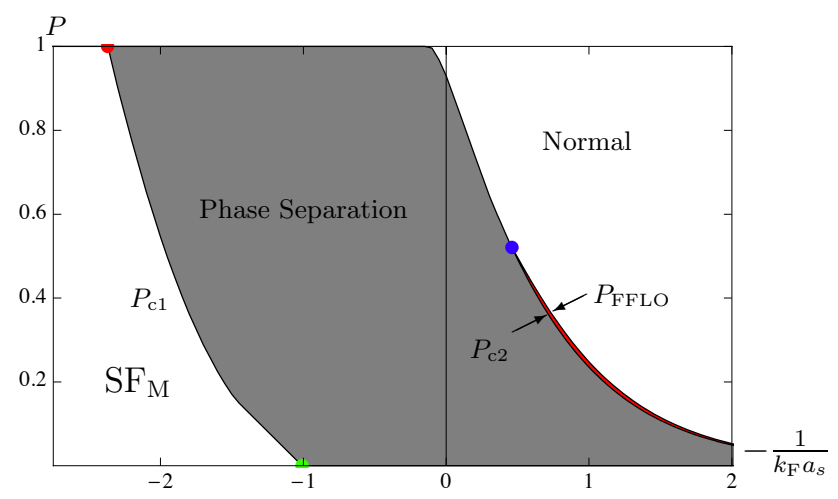

FIG. 1: A mean-field zero-temperature phase diagram from Refs 79, 100 of an imbalanced resonant Fermi gas, as a function of the inverse scattering length and normalized species imbalance $P=\left(N_{\uparrow}-N_{\downarrow}\right) / N \equiv \Delta N / N$, showing the magnetized (imbalanced) superfluid $\left(S F_{\mathrm{M}}\right)$, the FFLO state (approximated as the simplest FF state) confined to a narrow red sliver bounded by $P_{\mathrm{FFLO}}$ and $P_{\mathrm{c} 2}$, and the imbalanced normal Fermi liquid.

As illustrated in Fig 1, the key observation is that, despite strong interactions, within simplest mean-field treatments the conventional FFLO state 50,51 remains quite fragile, confined to a narrow sliver of polarization in the BCS regime [5, 79, 100, 131]. I emphasize that in fact above conclusion is only rigorously valid for the FF and not other forms (e.g., LO) of the FFLO class of 
states. Although the upper boundary, $h_{c 2}$, just below the normal state is trustworthy, as it is shared by all FFLO states, the lower one, $h_{c 1}$ can strongly depend on the form of the FFLO state, but was determined by Sheehy and Radzihovsky only for the FF state 79, 100, 133. Furthermore, motivated by earlier studies of the Bogoluibov-de Gennes (BdG) equation for the LO state 134 136], combined with finding of a negative domain-wall energy in an otherwise fully-paired singlet BCS superfluid in Zeeman field [136, 137], these studies have quite convincingly argued, that a more generic pair-density wave state (that includes a larger set of collinear wavevectors [118, 119]) may be significantly more stable. The quantitative ex-

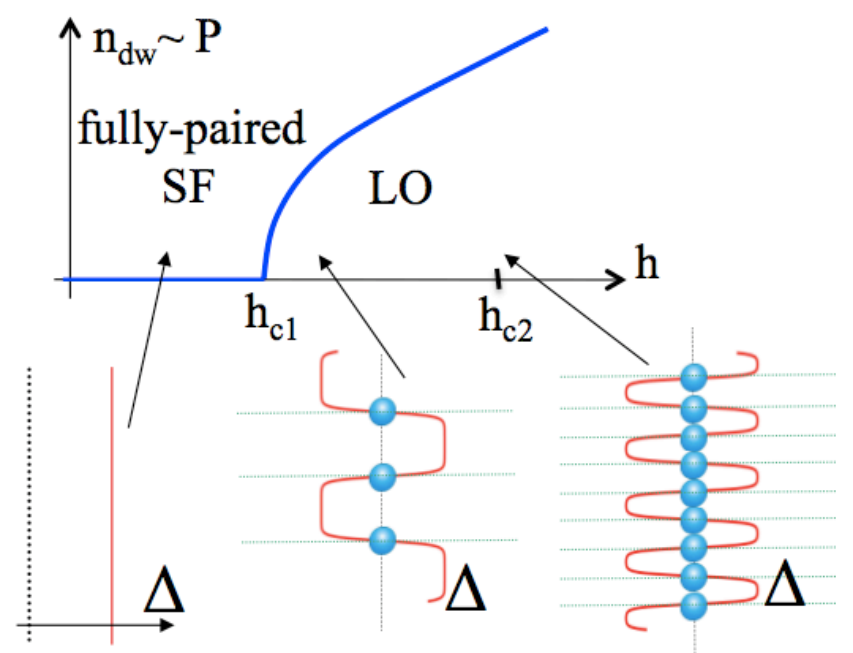

FIG. 2: An illustration of a continuous commensurateincommensurate (CI) transition at $h_{c 1}$ from a fullygapped (balanced) paired-superfluid to an imbalanced LarkinOvchinnikov superfluid. The excess majority atoms are localized on the domain walls in (zeros of) the LO order parameter, whose number $n_{d w}(h)$ is then proportional to the imbalance $P(h)$ and grows continuously with the chemical potential difference (Zeeman energy), $h-h_{c 1}$.

tent of the energetic stability of a PDW states in the imbalance-detuning phase diagram, in my view remains a widely open and urgent question. Consistent with above arguments but in absence of controlled quantitative analysis, I take the optimistic point of view that the LO states can extend over significantly wider region of the phase diagram, as schematically illustrated in Fig. 3 . Assuming it is indeed energetically stable, its phenomenology has been explored beyond its mean-field cartoon [50, 51] (latter only appropriate in the solid state and optical lattices, but not in the isotropically-trapped resonant atomic gases, where fluctuations are large).

\section{B. Summary}

Before turning to details, I summarize the salient features of the isotropically-trapped collinear class of FFLO

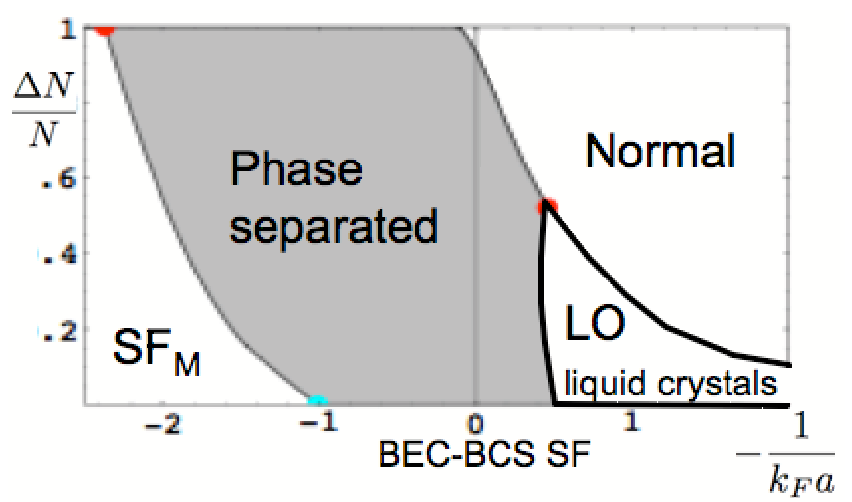

FIG. 3: A proposed $P=\Delta N / N$ vs. $1 /\left(k_{\mathrm{F}} a_{s}\right)$ phase diagram for an imbalanced resonant Fermi gas, showing the more stable LO liquid crystal phases (discussed in the text and illustrated in detail Fig 5 replacing a portion of the phaseseparated regime.

states, beyond their mean-field approximation. The key observation 66, 67] is that such striped states spontaneously break continuous rotational symmetry, and as a result exhibit phonon-like Goldstone modes that are qualitatively (energetically) softer than the solid-state analogs (where only discrete rotational symmetry can be broken) and all other superfluids. Namely, striped FF 138 and LO classes of states are characterized by highly anisotropic (with the modulation wavevector $\mathbf{q}_{0}=q_{0} \hat{z}$ and $\perp$ transverse to it) collective spectra, with

$$
\omega\left(k_{\perp}, k_{z}\right) \sim \sqrt{K k_{\perp}^{4}+B k_{z}^{2}},
$$

rather than by the usual linear-in-momentum Bogoluibov sound mode. The more experimentally relevant striped LO state, also exhibits a quantitatively anisotropic Bogoluibov linear-in- $k$ sound mode, but with the superfluid stiffness ratio $\rho_{\perp}^{s} / \rho_{z}^{s}$ that vanishes as the $h_{c 2}$ transition to the normal imbalanced Fermi gas is approached from below.

As a result, the fluctuations in such "soft" superfluid smectic states are qualitatively stronger. Although the states are stable to quantum fluctuations, in $3 \mathrm{~d}$ the LO and FF long-range orders are marginally unstable at any nonzero temperature. Consequently, (seemingly paradoxically) inside the LO state the average LO order parameter

$$
\begin{aligned}
\left\langle\Delta_{L O}(\mathbf{r})\right\rangle_{R} & =\left\langle 2 \Delta_{q_{0}} e^{i \phi(\mathbf{r})} \cos \left(\mathbf{q}_{0} \cdot \mathbf{r}+\theta(\mathbf{r})\right)\right\rangle_{R} \\
& \sim \frac{1}{R^{\eta}} \cos \mathbf{q}_{0} \cdot \mathbf{r} \longrightarrow 0
\end{aligned}
$$

vanishes in the thermodynamic limit (of a large cloud with atom number $N$ and cloud size $R \rightarrow \infty)$, suppressed to zero by thermal phonon $\theta / q_{0}$ fluctuations. The LO state is therefore strictly speaking homogeneous on long scales, exhibiting "algebraic topological", but no long-ranged translational order. Namely, the mean-field approximation fails qualitatively and the state instead 
is characterized by power-law order-parameter correlations, distinguished from the spatially short-ranged disordered phase by confined topological defects (bound dislocations), not by a nonzero LO order parameter. It is therefore a $3 \mathrm{~d}$ analog of the more familiar quasi-longrange ordered superfluid film, a $2 \mathrm{~d}$ easy-plane ferromagnet and a 2d crystal [139, 141 144].

As a consequence, a $3 \mathrm{~d}$ LO state is characterized by a static structure function $S(\mathbf{q})$ and momentum distribution function $n(\mathbf{k})$ with universal anisotropic quasi-Bragg peaks (around $q_{0}$ ), akin to the Landau-Peierls [139, 140. behavior of films of a conventional superfluid and $2 \mathrm{~d}$ crystals 141-144. Such novel behavior is not, however, exhibited by $3 \mathrm{~d}$ crystalline FFLO states with multiple non-collinear ordering wavevectors 118, 119, that, in contrast are characterized by the long-range positional order and a nonzero pair-condensate, that is stable to thermal fluctuations.

Another fascinating feature that arises because the LO order parameter, $\Delta_{L O}$ is a product (rather than the sum, as it is in a conventional supersolid) of the superfluid and density-wave component, is the unusual topological excitation that is a half-vortex bound to a half-dislocation in addition to integer vortices and dislocations.

In $2 \mathrm{~d}$, at nonzero $T$ the LO state is even more strongly disordered, characterized by short-range positional order with Lorentzian structure function peaks, and unstable to proliferation of dislocations 145. The state that results from such dislocated superfluid smectic is either a "charge"-4 (paired Cooper pairs) nematic superfluid 37, 66] or a nematic (possibly "fractionalized") Fermi liquid 35, 47, latter qualitatively the same as the deformed Fermi surface state [52.

Furthermore, a consideration of states that arise due to unbinding of various combination of topological defects (illustrated in the flow-chart in Fig 4 leads to a rich array of LO descendent states, that generically must intervene between the LO superfluid and a fully-paired conventional (isotropic and homogeneous) superfluid and a conventional polarized Fermi liquid. If indeed, as argued above, the $3 \mathrm{~d}$ LO state is energetically stable, these novel states are expected to appear in the region collectively denoted "LO liquid crystals" of the detuningpolarization phase diagram of Fig, 3 . They include a nonsuperfluid smectic $\left(F L_{S m}^{2 q}\right.$, driven by an unbinding of integer $2 \pi$-vortices), and a superfluid $\left(S F_{N}^{4}\right.$, driven by

\section{Microscopic model of imbalanced Fermi gas}

The physics of imbalanced atomic Fermi gases interacting through a broad Feshbach resonance is well captured by the one-channel model 3 [5, 146], characterized by a a proliferation of integer $a$-dislocations) and a nonsuperfluid $\left(F L_{N}\right.$, driven by an unbinding of both vortices and dislocations) nematics, and the corresponding isotropic states, when disclinations also condense. In addition, a variety of topologically-ordered isotropic and nematic "fractionalized" Fermi-liquid states $\left(F L_{N}^{*}, F L_{N}^{* *}, F L_{I}^{*}\right.$, and others) were predicted 66,67 , that are distinguished from their more conventional fully-disordered forms by gapped (bound) half-integer defects. These phases are summarized by a flowchart Fig 4 and a schematic phase diagram illustrated in Fig.5.

Finally, the fermionic sector of the LO gapless super-

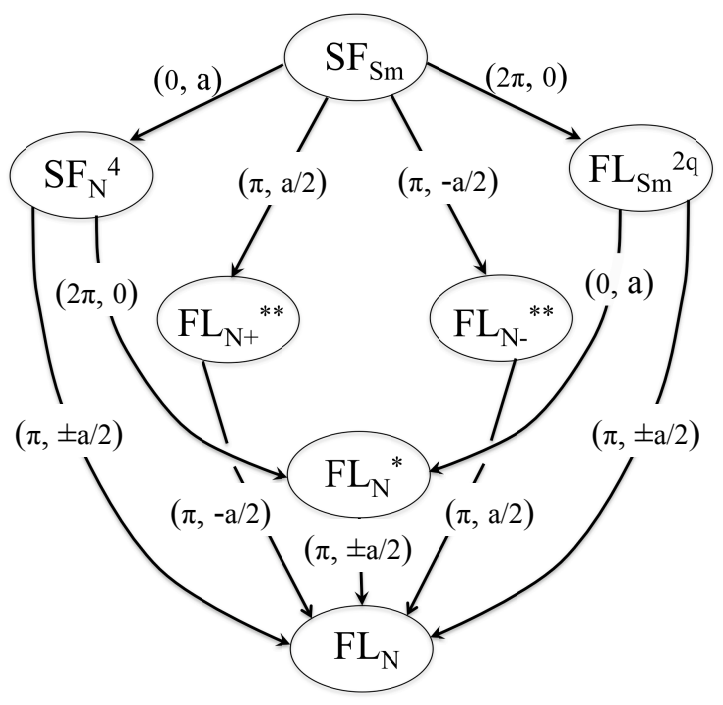

FIG. 4: A flowchart of superfluid $(S F)$ and nonsuperfluid $(F L)$ phases, exhibiting smectic $(S m)$ and nematic $(N)$ conventional orders as well as topological orders (indicated by $*$ and $* *)$, induced by a proliferation of various combination of topological defects, $(0, a),(2 \pi, 0)$, and $(\pi, \pm a / 2)$.

conductor is also quite unique, exhibiting a Fermi surface of Bogoliubov quasiparticles associated with the Andreev band of states, localized on the array of the LO domain walls. Consequences of the interplay between these fermionic and Goldstone mode degrees of freedom remains an open problem.

grand-canonical Hamiltonian

$$
H=\sum_{\mathbf{k}, \sigma}\left(\epsilon_{k}-\mu_{\sigma}\right) \hat{c}_{\mathbf{k} \sigma}^{\dagger} \hat{c}_{\mathbf{k} \sigma}+g \sum_{\mathbf{k q p}} \hat{c}_{\mathbf{k} \uparrow}^{\dagger} \hat{c}_{\mathbf{p} \downarrow}^{\dagger} \hat{c}_{\mathbf{k}+\mathbf{q} \downarrow} \hat{c}_{\mathbf{p}-\mathbf{q} \uparrow}
$$

with the single-particle energy $\epsilon_{k}=\hbar^{2} k^{2} / 2 m$. The separately conserved number $N_{\sigma}=\left(N_{\uparrow}, N_{\downarrow}\right)$ of atomic species (hyperfine states) $\sigma=\uparrow, \downarrow$ ) are imposed by two chemical potentials, $\mu_{\sigma}=\left(\mu_{\uparrow}, \mu_{\downarrow}\right)$. 


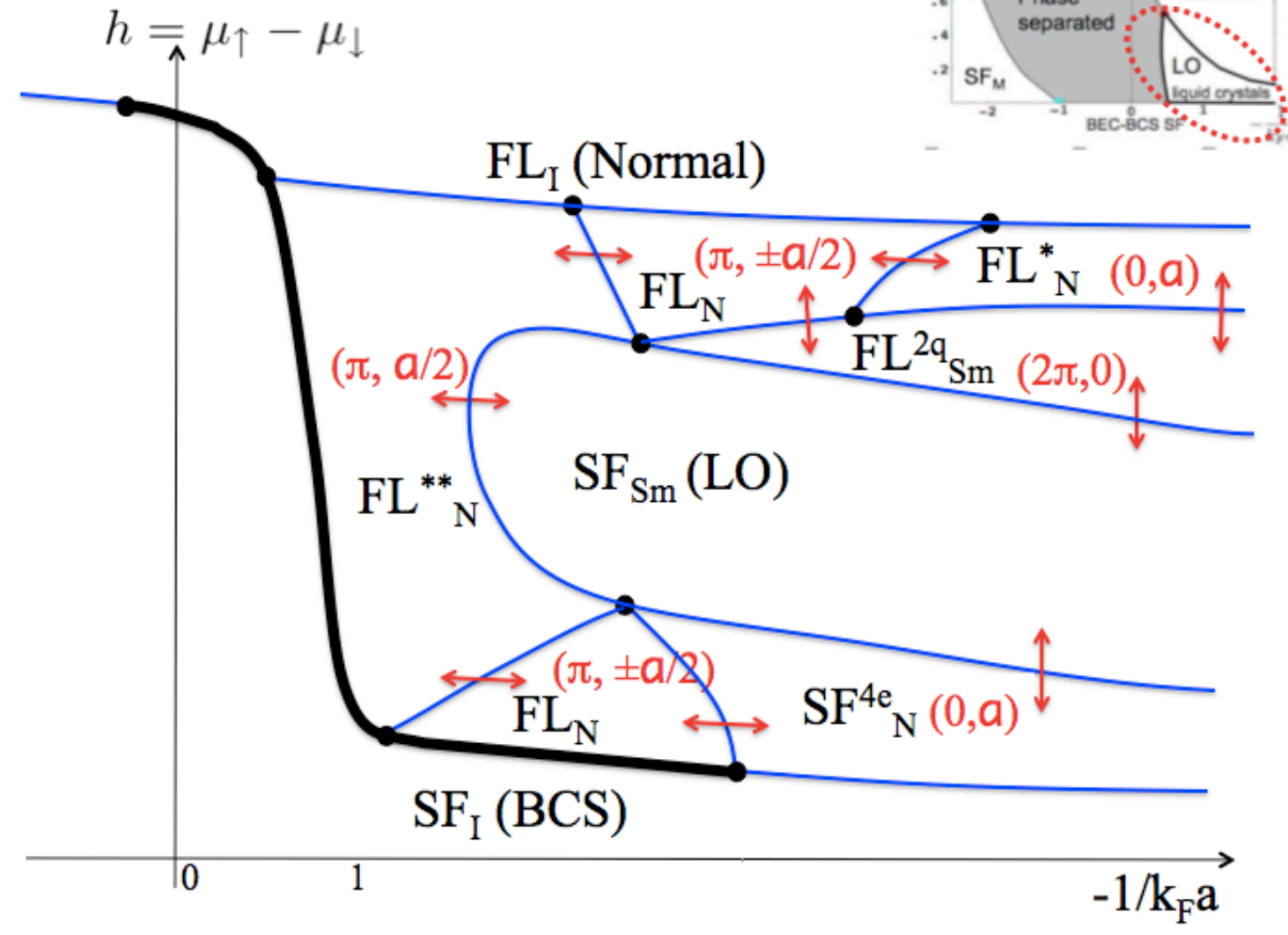

FIG. 5: A schematic imbalance-chemical potential (Zeeman energy), $h=\mu_{\uparrow}-\mu_{\downarrow}$ vs detuning (interaction strength), $-1 / k_{F} a$ phase diagram, illustrating the $3 \mathrm{~d}$ LO smectic phase $\left(S F_{S m}\right)$ and its descendant (described in the text), driven by a proliferation of various combinations of topological defects. The inset shows the global imbalance-interaction BCS-BEC phase diagram, illustrating the location of these putative phases.

The key feature that distinguishes this Fermi system from those familiar from solid state contexts is the attractive resonant interaction parameterized by a short-range s-wave pseudopotential, $g<0$. Through an exact Tmatrix scattering calculation $[3], g$ controls the magneticfield tunable [148, scattering length

$$
a_{s}(g)=\frac{m}{4 \pi} \frac{g}{1+g / g_{c}},
$$

that diverges above a critical attraction strength, $g_{c}=$ $\frac{2 \pi^{2}}{\Lambda m}(\Lambda \sim 1 / d$ is the short-scale pseudo-potential cutoff set by the extent of the molecular bound state), corresponding to a formation of a two-atom bound state.

The many-body thermodynamics of the resonant Fermi gas as a function of $N, \Delta N, T, a_{s}$, (i.e., the extension of the BEC-BCS crossover to a finite imbalance, $\Delta N)$ at large $k_{F}\left|a_{s}\right|$ presents a formidable challenge. However, much progress has been made in mapping out its qualitative (and in some regimes quantitative) phenomenology through a variety of approximate theoretical techniques, including quantum Monte Carlo [6], mean- field theory [79, 80, 100, 101, 103, the large- $N_{f}$ (fermion flavor) [109, 110] and $\epsilon$-expansions [107.

The simplest of these is the standard mean-field analysis 79 , 100 that gives a satisfactory qualitative description (quantitatively valid deep in the weakly-coupled BCS regime, $k_{F}\left|a_{s}\right| \ll 1$ ), as a starting point of more sophisticated treatments. To this end we assume the existence of a condensate

$$
\Delta(\mathbf{r})=\sum_{\mathbf{q}} \Delta_{\mathbf{q}} \mathrm{e}^{i \mathbf{q} \cdot \mathbf{r}}=g\left\langle\hat{c}_{\downarrow}(\mathbf{r}) \hat{c}_{\uparrow}(\mathbf{r})\right\rangle,
$$

corresponding to pair-condensation at momenta $\mathbf{q}$, with the set of amplitudes $\Delta_{\mathbf{q}}$ and $\mathbf{q}$ to be self-consistently determined by the minimizing the ground state energy subject to the constraints of fixed total atom number $N=N_{\uparrow}+N_{\downarrow}$ and the atom species number imbalance ("polarization") $\Delta N=N_{\uparrow}-N_{\downarrow}$, imposed by the average and difference chemical potentials $\mu, h=\frac{1}{2}\left(\mu_{\uparrow} \pm \mu_{\downarrow}\right)$, latter corresponding to the pseudo Zeeman energy.

Specializing to the simplest case of a single $\mathbf{q}$ of the Fulde-Ferrell state 50, this reduces the Hamiltonian to a 
quadratic Bogoluibov form, that can be easily diagonalized. This gives [79, 100] the ground state energy

$$
\begin{aligned}
E_{G S}^{F F}= & \sum_{\mathbf{k}}\left(\varepsilon_{k}-E_{k}+E_{\mathbf{k} \uparrow} \Theta\left(-E_{\mathbf{k} \uparrow}\right)+E_{\mathbf{k} \downarrow} \Theta\left(-E_{\mathbf{k} \downarrow}\right)\right) \\
& -\frac{1}{g}\left|\Delta_{\mathbf{q}}\right|^{2}
\end{aligned}
$$

and the excitation spectrum $E_{\mathbf{k} \sigma}$

$$
E_{\mathbf{k} \uparrow / \downarrow}=E_{k} \mp h \mp \frac{\mathbf{k} \cdot \mathbf{q}}{2 m},
$$

with $\varepsilon_{k} \equiv \frac{k^{2}}{2 m}-\mu+\frac{q^{2}}{8 m}$ and $E_{k} \equiv\left(\varepsilon_{k}^{2}+\Delta_{\mathbf{q}}^{2}\right)^{1 / 2}$. $E_{G S}^{F F}$ (and its generalizations to finite-temperature free energy 103 ), then gives all the thermodynamics, including the phase behavior summarized by the phase diagram in Fig.1.

In particular, this analysis predicts the existence of the FF state, stable only over a narrow sliver of imbalance, closing down for $-1 /\left(k_{F} a\right)>0.5$ [79, 100]. As mentioned in the Introduction, there are compelling arguments suggesting that this fragility is specific to the single q planewave FF condensate, and that the more generic PDW states are far more stable because they allow energetically important amplitude modulation [67, 134 137]. However, general PDW states are difficult to analyze at the transition from the fully gapped, balanced paired superfluid, near the lower critical field $h_{c 1}$ (at vanishing imbalance). In contrast, an analytic analysis near the upper-critical chemical potential difference $h_{c 2}$ at the transition from the normal state is indeed possible as a controlled Ginzburg-Landau expansion in the small pairdensity wave amplitude, $\Delta_{\mathbf{q}}$ [51, 66, 67, 147. While not quantitatively accurate away far below the $h_{c 2}$ transition (where PDW order parameter is large and is not limited to a single Fourier component $\Delta_{q}$ ) such Landau expansion is expected to be qualitatively correct and is a good starting point for a more complete analysis of fluctuations and phase transitions into the PDW LO state.

\section{Order-parameter theory of FFLO states}

\section{Ginzburg-Landau expansion near $h_{c 2}$}

The analytical treatment of the FFLO states near $h_{c 2}$ relies on the Ginzburg-Landau expansion in $\Delta_{\mathbf{q}}$, that is small near the (in mean-field) continuous $h_{c 2}$ normal-toFFLO transition [51, 149]. This expectation is supported by the exact $1 \mathrm{~d}$ BdG solution 134 at high fields, where $\Delta(x)$ is indeed well-approximated by a single sinusoid, with an amplitude $\Delta_{q}$ that vanishes continuously near $h_{c 2}$.

Consistent with these general arguments, by integrating out the atomic degrees of freedom, near $h_{c 2}$ the Ginzburg-Landau expansion for the ground-state energy takes a familiar form

$$
\mathcal{H} \approx \sum_{\mathbf{q}} \varepsilon_{q}\left|\Delta_{\mathbf{q}}\right|^{2}+\sum_{\left\{\mathbf{q}_{i}\right\}} V_{\mathbf{q}_{1}, \mathbf{q}_{2}, \mathbf{q}_{3}, \mathbf{q}_{4}} \Delta_{\mathbf{q}_{1}}^{*} \Delta_{\mathbf{q}_{2}} \Delta_{\mathbf{q}_{3}}^{*} \Delta_{\mathbf{q}_{4}}
$$

where the dispersion is given by [51, 67, 79, 100,

$$
\begin{aligned}
\varepsilon_{q} & \approx \frac{3 n}{4 \epsilon_{F}}\left[-1+\frac{1}{2} \ln \frac{v_{F}^{2} q^{2}-4 h^{2}}{\Delta_{B C S}^{2}}+\frac{h}{v_{F} q} \ln \frac{v_{F} q+2 h}{v_{F} q-2 h}\right], \\
& \approx J\left(q^{2}-q_{0}^{2}\right)^{2}+\varepsilon_{q_{0}},
\end{aligned}
$$

whose minimum at a finite $q_{0}(h) \approx 1.2 h / v_{F}$ (near $h_{c 2}$ ) captures the imbalanced atomic Fermi system's energetic tendency to pair at a finite momentum, and thereby to form a pair-density wave characterized by a reciprocal lattice vector with magnitude $q_{0}$ and a spontaneously chosen orientation. The value of $h$ at which $\varepsilon_{q_{0}}$ vanishes determines the corresponding mean-field N-FFLO transition point. While at quadratic order, all Fourier modes with $|\mathbf{q}|=q_{0}$ are degenerate, becoming unstable simultaneously, the form of the FFLO state is dictated by the interaction vertex function, $\tilde{V}_{\mathbf{q}_{1}, \mathbf{q}_{2}, \mathbf{q}_{3}, \mathbf{q}_{4}}$ that has been explicitly computed. Near the transition the physics of a unidirectional pair-density wave (Cooperpair stripe) order, characterized by a collinear set of $\mathbf{q}_{n}$ 's is well captured by focusing on long-wavelength fluctuations of these most unstable modes, well described by a Ginzburg-Landau Hamiltonian density

$\mathcal{H}=J\left[\left|\nabla^{2} \Delta\right|^{2}-2 q_{0}^{2}|\nabla \Delta|^{2}\right]+r|\Delta|^{2}+\frac{1}{2} \lambda_{1}|\Delta|^{4}+\frac{1}{2} \lambda_{2} \mathbf{j}^{2}$,

where deep in the BCS limit, near the $h_{c 2}$ the model parameters are given by

$$
\begin{aligned}
J & \approx \frac{0.61 n}{\epsilon_{F} q_{0}^{4}}, q_{0} \approx \frac{1.81 \Delta_{B C S}}{\hbar v_{F}}, r \approx \frac{3 n}{4 \epsilon_{F}} \ln \left[\frac{9 h}{4 h_{c 2}}\right], \\
h_{c 2} & \approx \frac{3}{4} \Delta_{B C S}, \quad \lambda_{1} \approx \frac{3 n}{4 \epsilon_{F} \Delta_{B C S}^{2}}, \quad \lambda_{2} \approx \frac{1.83 m^{2}}{\epsilon_{F} \Delta_{B C S}^{2} q_{0}^{2}},
\end{aligned}
$$

and the inclusion of the current-current interaction, $\mathbf{j}=\frac{1}{m} \operatorname{Re}\left[-\Delta^{*}(\mathbf{r}) i \nabla \Delta(\mathbf{r})\right]$ is necessary for a complete description. More generally, away from the weak-coupling BCS limit these couplings can be taken as phenomenological parameters to be determined experimentally, but the general form of the Ginzburg-Landau model has broader range of applicability, capturing all the qualitative features of the transition and the PDW state.

\section{Larkin-Ovchinnikov state near $h_{c 1}$}

However, the derivation and expressions for the associated couplings, Eqs. (11) are limited to the weak coupling BCS regime and near the high chemical potential imbalance (Zeeman field) normal-to-FFLO transition at $h_{c 2}$.

In a complementary, low chemical potential imbalance regime, just above the transition from the fully-paired 
(BCS-BEC) superfluid to the LO state at $h_{c 1}$, a phenomenological analysis is possible 67. It treats the LO state as a periodic array of fluctuating $\pm \Delta$ domain-walls (stripes) in $\Delta(\mathbf{r})$, akin to the lyotropic phases in soft condensed matter 48, 49].

However, such approach implicitly assumes that as the domain-wall surface energy becomes negative 134 137. for $h>h_{c 1}$, their interaction remains repulsive, and so the domain-walls proliferate continuously as a periodic array inside the LO state. Under this assumption (that warrants further study) the domain-wall density $n_{d w}$ and the associated species imbalance $P \propto n_{d w}\left(\approx q_{0}(h)\right)$ is then set by a balance between the negative surface energy and the domain-wall repulsion, growing continuously as a function of $h-h_{c 1}$ according to the PokrovskyTalapov's commensurate-incommensurate (CI) transition phenomenology [150]. This behavior is clearly exhibited in 1d[134, 152, 153, through an exact solution and bosonization methods, and has been argued to persist in higher dimensions 134 137. The CI route for a transition to the LO state contrasts sharply with the Landau theory [51, 79, 100] of two independent order parameters $\Delta_{0}, \Delta_{q}$, that always predicts a first-order BCS$\mathrm{LO}$ transition. The latter corresponds to the case of an attractive domain-wall interaction, that therefore proliferate discontinuously above $h_{c 1}$, leading to the ubiquitous phase separation found in mean-field theory [79, 100]. It is currently unclear what dimensionless microscopic parameter, analogous to Abrikosov's $\kappa$ (distinguishing between type I and type II superconductors) 154, 155, controls these two alternatives of the macro-phase separation (a first-order transition) and the micro-phase separated LO state (a continuous transition out of the gapped SF state) [149. A detailed analysis of such low-imbalance approach to the SF-LO transition and the LO state is sorely missing and is a subject of current research.

A semi-phenomenological local density approximation (LDA) model that assembles all known ingredients is given by

$$
H[\Delta(\mathbf{r})] \simeq \int_{\mathbf{r}}\left[\frac{J}{2}\left(\left|\nabla^{2} \Delta\right|^{2}-2 q_{0}^{2}|\nabla \Delta|^{2}\right)+V(\Delta(\mathbf{r}))\right],
$$

where $\Delta_{0}=e^{1 / 2} \Delta_{B C S}$ and $V(\Delta)=-2 \nu(\mu) \Delta^{2} \ln \left(\frac{\Delta}{\Delta_{0}}\right)$ $\nu(\mu)\left[h \sqrt{h^{2}-\Delta^{2}}-\Delta^{2} \cosh ^{-1}(h / \Delta)\right] \Theta(h-\Delta)$ is the effective potential derived within a $\mathrm{BdG}$ analysis for a uniform $\Delta$. It fully captures the double-minimum structure and the associated 1st-order normal to (fully-gapped BCS) superfluid transition that skips the interesting intermediate e.g., the FFLO states. This LDA potential is supplemented by the gradient energy functional, inherited from a microscopic GL analysis (valid only near $h_{c 2}$ ). Its use near $h_{c 1}$ is supported by the fact that it is the simplest form that incorporates the underlying symmetries and encodes the expected energetics for the system to order at a finite $h$-dependent momentum even near $h_{c 1}$. Work is under way to derive this functional through a

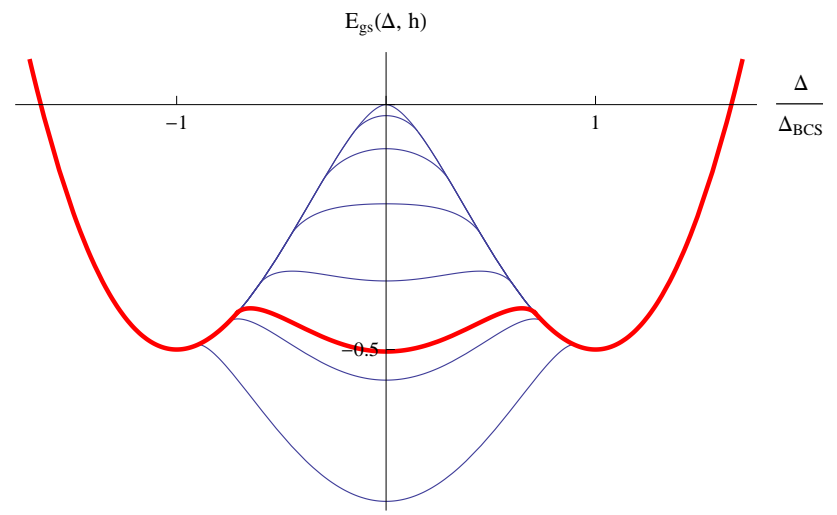

FIG. 6: Ground state energy $E_{g s}[\Delta, h]$ as a function of the order parameter $\Delta$ and Zeeman energy $h$, indicating a firstorder transition at $h_{c}$ (thick red curve) between the paired superfluid state $\left(\Delta_{B C S}\right)$ and the normal state $(\Delta=0)$, at fixed density and/or imbalance exhibiting phase-separated coexistence. As argued in the text, analogously to the PokrovskyTalapov systems and type-II superconductors, this first-order transition can be preempted by a continuous CI-like transition to a striped LO superfluid and its descendent quantum liquid-crystal states, illustrated in Fig, 2 .

controlled Moyal (semi-classical) gradient expansion on the BdG Hamiltonian.

The functional $E_{G}[\Delta(\mathbf{r})]$ has a double-well structure, with an additional normal state minimum (at $\Delta=0$ ) developing for $h>\Delta_{B C S} / 2$. It thus allows periodic soliton structure in $\Delta(\mathbf{r})$, corresponding to oscillations between the minima at $\pm \Delta_{B C S}$.

\section{E. Goldstone modes in striped FFLO states}

Using the Ginzburg-Landau model one can develop a low-energy Goldstone modes description of the striped paired states and use it to analyze their stability to fluctuations. We focus on the unidirectional (striped) pairdensity wave states, with FF and LO states as simplest representatives of two qualitatively distinct universality classes. The corresponding order parameter is given by

$$
\Delta_{F F L O}(\mathbf{r})=\Delta_{+}(\mathbf{r}) e^{i \mathbf{q} \cdot \mathbf{r}}+\Delta_{-}(\mathbf{r}) e^{-i \mathbf{q} \cdot \mathbf{r}},
$$

where $\Delta_{ \pm}(\mathbf{r})$ are two complex scalar order parameters, the dominant Fourier coefficients $\Delta_{ \pm}(\mathbf{r})=\Delta_{ \pm}^{0}(\mathbf{r}) e^{i \phi_{ \pm}(\mathbf{r})}$ and amplitudes $\Delta_{ \pm}^{0}$ distinguishing between the $\mathrm{FF}$ and LO states. Using this representation inside $\mathcal{H}$ and minimizing the ground-state energy for $h<h_{c 2}$ a simple analysis shows that indeed it is the LO state with $\Delta_{+}=\Delta_{-} \neq 0$ that is most stable inside the BCS regime; the FF state is characterized by only one nonvanishing order parameter, $\Delta_{+} \neq 0, \Delta_{-}=0[51]$. More generally, either state can be stabilized depending on the relative magnitudes of $\lambda_{1}$ and $\lambda_{2}$. 


\section{Fulde-Ferrell state}

The FF state is characterized by a single (independent) nonzero complex order parameter,

$$
\Delta_{F F}(\mathbf{r})=\Delta_{q_{0}} e^{i \mathbf{q}_{0} \cdot \mathbf{r}+i \phi},
$$

that is a plane-wave with the momentum $\mathbf{q}_{0}$ and a single Goldstone mode $\phi=\phi_{+}$corresponding to the local superconducting phase. The state carries a nonzero, uniform spontaneously-directed supercurrent $\mathbf{j}_{F F}=\frac{1}{m}\left|\Delta_{q_{0}}\right|^{2}\left(\mathbf{q}_{0}+\boldsymbol{\nabla} \phi\right)$ and thereby breaks the timereversal and rotational symmetry, chosen spontaneously along $\mathbf{q}_{0}$, as well as the global gauge symmetry, corresponding to the total atom conservation. Although the FF order parameter itself is not translationally invariant, it is invariant under a modified transformation of an arbitrary translation followed by a gauge transformation, with all gauge-invariant observables thus translationally invariant. Thus, the FF state is a uniform orientationally-ordered polar superfluid. The underlying rotational invariance also demands that it is invariant under a rotation of $\mathbf{q}_{0}=q_{0} \hat{z}$ by an angle $\alpha$ that generates a nontrivial, spatially-dependent phase $\phi^{0}(\mathbf{r})=z(\cos \alpha-1)+x \sin \alpha$. Simple algebra demonstrates that the fully nonlinear form of the longitudinal current $\delta j_{\|}=\partial_{\|} \phi+\frac{1}{2} q_{0}^{-1}(\nabla \phi)^{2}$ ensures that it and the corresponding energy $\mathcal{H}_{F F}$ vanish for $\phi^{0}(\mathbf{r})$, as required by the underlying rotational invariance.

The analysis of the GL functional lead to a Goldstone mode $\phi$ Hamiltonian 66, 67, 138.

$$
\mathcal{H}_{F F}=\frac{1}{2} K\left(\nabla^{2} \phi\right)^{2}+\frac{1}{2} \rho_{s}^{\|}\left(\partial_{\|} \phi+\frac{1}{2} q_{0}^{-1}(\nabla \phi)^{2}\right)^{2},
$$

where $\partial_{\|} \equiv \hat{\mathbf{q}}_{0} \cdot \nabla, \rho_{s}^{\|}=8 J q^{2}\left|\Delta_{q_{0}}\right|^{2}$ is the superfluid stiffness along $q_{0}$ and $K=2 J\left|\Delta_{q_{0}}\right|$. The Hamiltonian form, $\mathcal{H}_{F F}$ is valid beyond its weak-coupling microscopic derivation and is familiar from studies of conventional smectic liquid crystals 48, 49, 156, despite the fact that FF state is a translationally-invariant polar superfluid not a smectic. The necessity of keeping the higher order gradients and $\delta j_{\|}$nonlinearities in $\mathcal{H}_{F F}$ is due to the identical vanishing of the transverse superfluid stiffness, $\rho_{s}^{\perp}=0$ (guaranteed by the underlying rotational invariance unique to the FFLO striped states, absent in solid state contexts) that leads to fluctuations that are otherwise infrared-divergent in a purely harmonic model.

\section{Larkin-Ovchinnikov state}

The LO state is instead described by two independent nonzero PDW amplitudes $\Delta_{+}=\Delta_{-} \equiv \Delta_{q_{0}}$ (growing below $h_{c 2}$ ), that lead to a standing wave pair-density wave order parameter,

$$
\begin{aligned}
\Delta_{L O}(\mathbf{r}) & =2 \Delta_{q_{0}} e^{i \frac{1}{2}\left(\phi_{+}+\phi_{-}\right)} \cos \left[\mathbf{q}_{0} \cdot \mathbf{r}+\frac{1}{2}\left(\phi_{+}-\phi_{-}\right)\right] \\
& =2 \Delta_{q_{0}} e^{i \phi} \cos \left[\mathbf{q}_{0} \cdot \mathbf{r}+\theta\right]
\end{aligned}
$$

that is a product of a superfluid and a unidirectional density wave striped order parameters. These are respectively characterized by two Goldstone modes $\phi, \theta$, corresponding to the superfluid phase and the smectic phonon $u=-\theta / q_{0}$ of the striped state. This also contrasts with the conventional smectic 48. (e.g., in liquid crystal materials, where one instead is dealing with a real mass density $\rho(\mathbf{r})$ not a pair condensate wavefunction), characterized by a single phonon Goldstone mode, $u$.

The mean-field LO order parameter, $\Delta_{L O}$ simultaneously exhibits the ODLRO (superfluid) and the smectic (unidirectional density wave) orders. It thus spontaneously breaks the rotational, translational, and global gauge symmetries, and therefore realizes a form of a paired supersolid. However, it is distinguished from a conventional purely bosonic supersolid 125 128, where homogeneous superfluid order and periodic density wave coexist, by the vanishing of the ("charge"-2 two-atom) zero momentum $(\mathbf{q}=0)$ superfluid component in the LO condensate[129].

Similarly to the FF state, the underlying rotational symmetry of the LO state strongly restricts the form of the Goldstone-mode Hamiltonian. Namely, its $\theta=-q_{0} u$ sector must be invariant under a rotation of $\mathbf{q}_{0}$, that defines the spontaneously-chosen orientation of the pairdensity wave, and therefore must be described by a smectic form 48, 49, 156. On the other hand because a rotation of the LO state leaves the superconducting phase, $\phi$ unchanged, the superfluid phase $\phi$ sector of the Hamiltonian is therefore expected to be of a conventional $x y$ model type. Consistent with these symmetry-based expectations the LO Goldstone-mode Hamiltonian was indeed found 66, 67, to be given by

$$
\begin{aligned}
\mathcal{H}_{L O}= & \frac{1}{2} K\left(\nabla^{2} u\right)^{2}+\frac{1}{2} B\left(\partial_{\|} u-\frac{1}{2}(\nabla u)^{2}\right)^{2} \\
& +\frac{1}{2} \rho_{s}^{\|}\left(\partial_{\|} \phi\right)^{2}+\frac{1}{2} \rho_{s}^{\perp}\left(\nabla_{\perp} \phi\right)^{2},
\end{aligned}
$$

with the nonlinear strain tensor $u_{q q}=\hat{\mathbf{q}} \cdot \nabla u-\frac{1}{2}(\nabla u)^{2}$ ensuring the full rotational invariance. In the weaklycoupled BCS limit the smectic elastic moduli and the superfluid stiffnesses are given by

$$
\begin{aligned}
K & =4 J q_{0}^{2}\left|\Delta_{q_{0}}\right|^{2} \approx \frac{0.8 n \Delta_{B C S}^{2}}{\epsilon_{F} q_{0}^{2}} \ln \left(h / h_{c 2}\right), \\
B & =16 J q_{0}^{4}\left|\Delta_{q_{0}}\right|^{2} \approx \frac{3.3 n \Delta_{B C S}^{2}}{\epsilon_{F}} \ln \left(h / h_{c 2}\right), \\
\rho_{s}^{\|} & =B / q_{0}^{2} \\
\rho_{s}^{\perp} & =\frac{4 \lambda_{2}}{m^{2}}\left|\Delta_{q_{0}}\right|^{4} \approx \frac{0.8 n \Delta_{B C S}^{2}}{\epsilon_{F} q_{0}^{2}} \ln ^{2}\left(h / h_{c 2}\right) .
\end{aligned}
$$

Thus, the LO state is a highly anisotropic superfluid (though less so than the FF state, where $\rho_{s}^{\perp}=0$ ), with

$$
\frac{\rho_{s}^{\perp}}{\rho_{s}^{\|}}=\frac{3}{4}\left(\frac{\Delta_{q_{0}}}{\Delta_{B C S}}\right)^{2} \approx \frac{1}{4} \ln \left(\frac{h_{c 2}}{h}\right) \ll 1,
$$


a ratio that vanishes for $h \rightarrow h_{c 2}^{-}$.

We stress that while the detailed expressions for the moduli above are specific to the weak-coupling BCS limit near $h_{c 2}$ the general form of $\mathcal{H}_{L O},(17)$, including the structure of the symmetry-enforced nonlinearities in the $u$ (smectic) sector is valid beyond our microscopic derivation, and holds throughout the LO phase.

By extending the Hamiltonian to include density fluctuations, $\delta n_{ \pm}$, canonically conjugate to $\phi_{ \pm}$and integrating them out in an imaginary time $(\tau)$ coherent-state path integral, leads to a Lagrangian density

$$
\mathcal{L}=\frac{\chi_{0}}{2}\left(\partial_{\tau} \phi_{+}\right)^{2}+\frac{\chi_{0}}{2}\left(\partial_{\tau} \phi_{-}\right)^{2}+\mathcal{H}\left[\phi_{+}, \phi_{-}\right],
$$

with $\mathcal{H}$ given by the $\mathcal{H}_{L O}$ in the $\mathrm{LO}$ ground state $(\mathrm{FF}$ state is treated similarly using $\mathcal{H}_{F F}$ and a single Goldstone mode). For the LO state, this analysis then predicts the existence of two anisotropic low-frequency modes with dispersions

$$
\begin{aligned}
& \omega_{\phi}(\mathbf{k})=\sqrt{\left(\rho_{s}^{\perp} k_{\perp}^{2}+\rho_{s}^{\|} k_{z}^{2}\right) / \chi_{0}}, \\
& \omega_{u}(\mathbf{k})=\sqrt{\left(K k_{\perp}^{4}+B k_{z}^{2}\right) / \chi_{0}},
\end{aligned}
$$

where $\chi_{0}$ is the compressibility of the Fermi gas. These modes respectively correspond to the zeroth sound (the Bogoliubov mode as in a conventional superfluid) and smectic phonon, unique to the LO state. In cold atomic gases, these should in principle be measurable via the Bragg spectroscopy technique 157-159.

With the Goldstone-mode Lagrangian in hand, the effects of quantum and thermal fluctuations as well as equilibrium correlation and response functions can be calculated 160 .

\section{Goldstone modes fluctuations}

Armed with the action for the Goldstone modes, the low-energy quantum and thermal fluctuations in the FFLO striped states are straightforwardly computed. Despite smectic like softness of these modes, it is easy to show that quantum fluctuations remain finite for $d>1$ and therefore the FF and $\mathrm{LO}$ states remain stable at zero temperature.

Harmonic approximation:

In contrast, at finite temperature the root-meansquared fluctuations of the smectic phonon modes in the LO state (and phase fluctuations in the FF state) diverge with trap size (in a purely harmonic Goldstone-modes theory) according to

$$
\left\langle u^{2}\right\rangle_{0}^{T} \approx \begin{cases}\frac{T}{2 \sqrt{B K}} L_{\perp}^{3-d}, & d<3 \\ \frac{T}{4 \pi \sqrt{B K}} \ln q_{0} L_{\perp}, & d=3\end{cases}
$$

where the fluctuations are evaluated in a trap with an aspect ratio $L_{\perp} \times L_{z}$, with $L_{z}$ its extent along the ordering $\mathbf{q}_{0}$ axis $48,49,162$. These fluctuations lead to an emergence of important crossover length scales $\xi_{z}, \xi_{\perp} \sim\left(\xi_{z} \sqrt{K / B}\right)^{1 / 2} \equiv \sqrt{\xi_{z} \lambda}$ that characterize the finite-temperature LO state,

$$
\xi_{\perp} \approx \begin{cases}\frac{a^{2} \sqrt{B K}}{T} \sim \frac{K}{T q_{0}}, & d=2, \\ a e^{4 \pi a^{2} \sqrt{B K} / T} \sim a e^{\frac{c K}{T q_{0}}}, & d=3,\end{cases}
$$

defined as scales at which LO phonon fluctuations are comparable to LO stripe period $a=2 \pi / q_{0}$.

The thermal connected correlation function of $\mathrm{LO}$ phonons

$$
C_{u}\left(\mathbf{r}_{\perp}, z\right)=\left\langle\left[u\left(\mathbf{r}_{\perp}, z\right)-u(\mathbf{0}, 0)\right]^{2}\right\rangle_{0}
$$

is also straightforwardly worked out, in $3 \mathrm{~d}$ giving the logarithmic Caillé form[162]

$$
C_{u}^{3 d}\left(\mathbf{r}_{\perp}, z\right) \approx \frac{T}{2 \pi \sqrt{K B}}\left\{\begin{array}{ll}
\ln \left(\frac{r_{\perp}}{a}\right), & r_{\perp} \gg \sqrt{\lambda|z|} \\
\ln \left(\frac{4 \lambda z}{a^{2}}\right), & r_{\perp} \ll \sqrt{\lambda|z|}
\end{array} .\right.
$$

In $2 \mathrm{~d}$ it is instead given by 145

$$
C_{u}^{2 d}(x, z) \approx \frac{2 T}{B}\left\{\begin{array}{ll}
\left(\frac{|z|}{4 \pi \lambda}\right)^{1 / 2}, & x \ll \sqrt{\lambda|z|} \\
\frac{|x|}{4 \lambda}, & x \gg \sqrt{\lambda|z|}
\end{array} .\right.
$$

Above finding of the divergence of smectic phonon fluctuations at nonzero temperature have immediate drastic implications for the properties of the $\mathrm{LO}$ (and FF) states. The most important of these is that the thermal average of the Landau's LO order parameters (16) vanishes in thermodynamic limit

$$
\left\langle\Delta_{L O}(\mathbf{r})\right\rangle_{0} \approx 2 \tilde{\Delta}_{q_{0}}\left(L_{\perp}\right) \cos \left(\mathbf{q}_{0} \cdot \mathbf{r}\right),
$$

with the thermally suppressed order parameter amplitude given by

$$
\begin{aligned}
\tilde{\Delta}_{q_{0}}\left(L_{\perp}\right) & =\Delta_{q_{0}} e^{-\frac{1}{2} \phi_{r m s}^{2}} \begin{cases}e^{-L_{\perp} / \xi_{\perp}}, & d=2, \\
\left(\frac{a}{L_{\perp}}\right)^{\eta / 2}, & d=3,\end{cases} \\
& \rightarrow 0, \text { for } L_{\perp} \rightarrow \infty,
\end{aligned}
$$

where $\phi_{r m s}^{2} \equiv\left\langle\phi^{2}\right\rangle_{0}$ accounts for the finite quantum and thermal superconducting phase fluctuations, and $\eta=\frac{q_{0}^{2} T}{8 \pi \sqrt{B K}}$ is the Caillé exponent 162 .

Akin to $2 \mathrm{~d}$ xy-model systems, the vanishing of the LO order parameter does not imply the instability of the phase, but that the true fluctuating state contrasts qualitatively with perfectly periodically-ordered mean-field description.

These divergent LO phonon fluctuations also qualitatively modify the Cooper-pair momentum distribution function $n_{\mathbf{k}}=\left\langle\Delta_{\mathbf{k}}^{\dagger} \Delta_{\mathbf{k}}\right\rangle$ and structure function $S(\mathbf{q})$ (respectively measurable via time-of-flight and Bragg spectroscopy [157-159] imaging of pair-condensate) from their true Bragg ( $\delta$-function peaks) form characteristic of the mean-field long-range periodic order. They are highly 


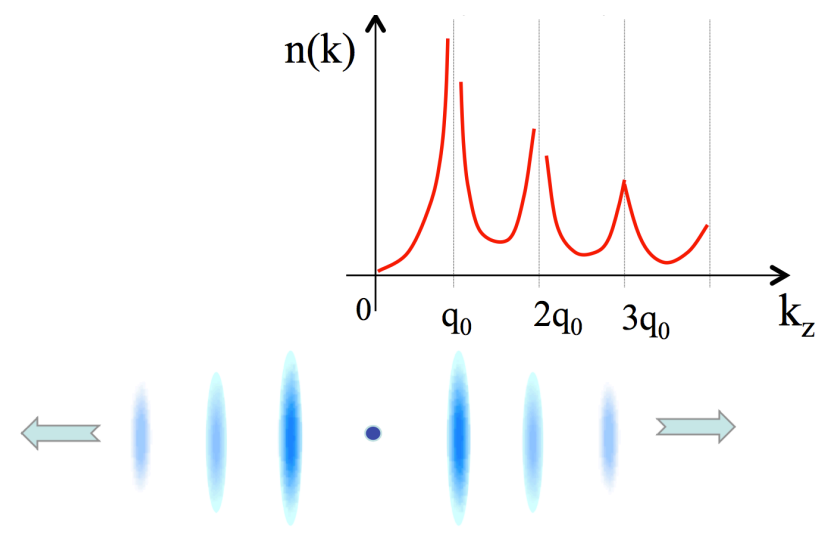

FIG. 7: The finite momentum pairing at $q_{0}$ and divergent $3 \mathrm{~d}$ smectic phonon fluctuations in the LO state are reflected in the Cooper-pair center-of-mass momentum distribution function, $n_{\mathbf{k}}$ (observable via the time-of-flight measurements), displaying power-law Bragg peaks, characteristic of spatial quasilong-range order.

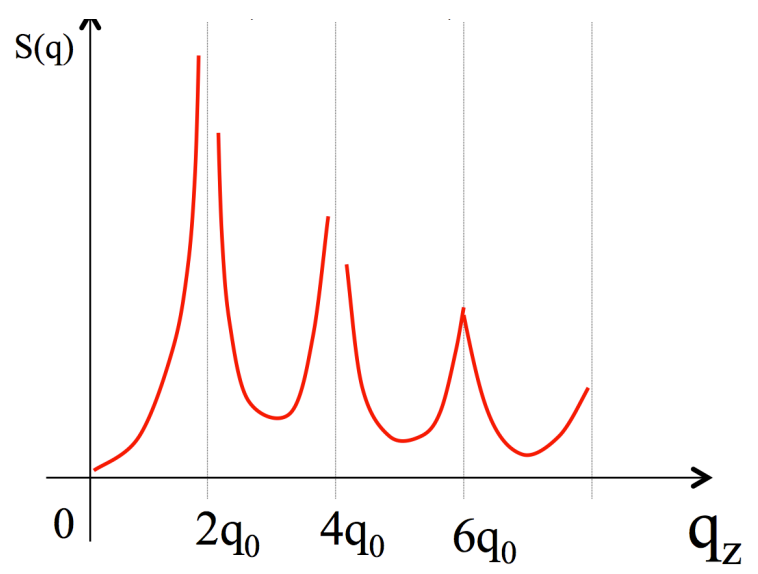

FIG. 8: The structure function, $S(\mathbf{q})$ for the $3 \mathrm{~d}$ LO state, displaying power-law Bragg peaks, characteristic of the LO superfluid's spatial quasi-long-range order.

anisotropic $\left(q_{z} \sim q_{\perp}^{2} / \lambda\right)$ and exhibit quasi-Bragg peaks (see Fig, 8) around the ordering wavevector $\mathbf{q}_{0}$ (and its harmonics, $\left.\mathbf{q}_{n}\right)$, reminiscent of $(1+1) d$ Luttinger liquids and two-dimensional crystals 139 144

$$
\begin{aligned}
n_{k_{z}}^{L O} & \approx \sum_{n} \frac{n_{q_{n}}}{\left|k_{z}-n q_{0}\right|^{2-n^{2} \eta}}, \\
S^{L O}\left(q_{z}\right) & \approx \sum_{n} \frac{\left|\Delta_{q_{n}}\right|^{4}}{\left|q_{z}-2 n q_{0}\right|^{2-4 n^{2} \eta}},
\end{aligned}
$$

These predictions are a reflection of the well-known 48 , 49] and experimentally tested 163 behavior of conventional smectic liquid crystals. In two dimensions, the
LO order is even more strongly suppressed by thermal fluctuations down to short-ranged correlations, strongly suggesting true instability of the LO order. Because it is the "soft" smectic Goldstone mode that is responsible for these interesting properties they are necessarily also shared by the FF state 138 .

I emphasize that analogous Kosterlitz-Thouless phase fluctuation physics has been observed in conventional $2 \mathrm{~d}$ trapped superfluids, despite the finite trap size [164, 165]. Thus, I am hopeful that they can be similarly seen in the 3d LO state. A more detailed analysis of the trap effects is necessary for direct comparison with experiments.

Another fascinating consequence of the vanishing of $\left.\left\langle\Delta_{L O}\right\rangle, \sqrt{28}\right\rangle$ is that the leading nonzero Landau order parameter characterizing the LO state is the translationally-invariant "charge"-4 (4-atom pairing) superconducting order parameter,

$$
\begin{aligned}
\Delta_{s c}^{(4)} & \sim \int_{\mathbf{r}, \mathbf{r}^{\prime}}\left\langle\hat{c}_{\downarrow}(\mathbf{r}) \hat{c}_{\uparrow}(\mathbf{r}) \hat{c}_{\downarrow}\left(\mathbf{r}^{\prime}\right) \hat{c}_{\uparrow}\left(\mathbf{r}^{\prime}\right)\right\rangle e^{i \mathbf{q} \cdot\left(\mathbf{r}-\mathbf{r}^{\prime}\right)}, \\
& \sim \sum_{\mathbf{k}, \mathbf{k}^{\prime}}\left\langle\hat{c}_{\downarrow,-\mathbf{k}} \hat{c}_{\uparrow, \mathbf{k}+\mathbf{q}} \hat{c}_{\downarrow,-\mathbf{k}^{\prime}} \hat{c}_{\uparrow, \mathbf{k}^{\prime}-\mathbf{q}}\right\rangle .
\end{aligned}
$$

Thus in the presence of thermal fluctuations the LO phase corresponds to an exotic state in which the offdiagonal order is exhibited by pairs of Cooper pairs, i.e., a bound quartet of atoms, rather than by the conventional 2-atom Cooper pairs 37, 66. In 2d and 3d this higher order pairing is driven by arbitrarily low- $T$ fluctuation, rather than by a fine-tuned attractive interaction between Cooper pairs, and therefore has no simple meanfield description. A microscopic formulation of such a state and its detailed properties remain an open problem.

\section{Nonlinear elasticity beyond Gaussian fluctuations:}

As discussed in the context of conventional smectics 156 and more recently for the FFLO state 66, 67, above predictions neglect the effects of Goldstone mode nonlinearities $\mathcal{H}_{\text {nonlinear }}=$ $-\frac{1}{2} B\left(\partial_{z} u\right)(\nabla u)^{2}+\frac{1}{8} B(\nabla u)^{4}$, in Eq. $\sqrt{15}$, (17), that modify the asymptotics on scales longer than the crossover scales $\xi_{\perp}^{N L}=\sqrt{\xi_{z}^{N L} \lambda}$,

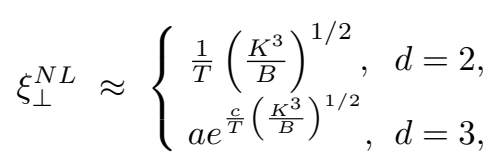

The behavior on scales beyond $\xi_{\perp, z}^{N L}$ can be obtained using renormalization-group analysis for $d \leq 3[67$, 156, with an exact solution in $2 \mathrm{~d}[166]$. The finite-temperature asymptotics is well-approximated by a correlation function

$$
G_{u}(\mathbf{k}) \approx \frac{T}{B(\mathbf{k}) k_{z}^{2}+K(\mathbf{k}) k_{\perp}^{4}},
$$

with moduli $B(\mathbf{k})$ and $K(\mathbf{k})$ that display a universal singular wavevector-dependence, that is asymptotically $e x$ - 
act logarithmic [156] in $3 \mathrm{~d}$

$$
\begin{aligned}
& K\left(\mathbf{k}_{\perp}, k_{z}=0\right) \sim K\left|1+\frac{5 g}{64 \pi} \ln \left(1 / k_{\perp} a\right)\right|^{2 / 5} \\
& B\left(\mathbf{k}_{\perp}=0, k_{z}\right) \sim B\left|1+\frac{5 g}{128 \pi} \ln \left(\lambda / k_{z} a^{2}\right)\right|^{-4 / 5} .
\end{aligned}
$$

and power-law in $2 \mathrm{~d}$

$$
\begin{aligned}
K(\mathbf{k}) & =K\left(k_{\perp} \xi_{\perp}^{N L}\right)^{-\eta_{K}} f_{K}\left(k_{z} \xi_{z}^{N L} /\left(k_{\perp} \xi_{\perp}^{N L}\right)^{\zeta}\right) \\
& \sim k_{\perp}^{-\eta_{K}} \\
B(\mathbf{k}) & =B\left(k_{\perp} \xi_{\perp}^{N L}\right)^{\eta_{B}} f_{B}\left(k_{z} \xi_{z}^{N L} /\left(k_{\perp} \xi_{\perp}^{N L}\right)^{\zeta}\right) \\
& \sim k_{\perp}^{\eta_{B}} .
\end{aligned}
$$

with $f_{B}(x), f_{K}(x)$ universal scaling functions and $\eta_{B}^{2 d}=$ $1 / 2, \eta_{K}^{2 d}=1 / 2, \zeta^{2 d}=3 / 2$ exact [166]. In $3 d$ this translates into an equal-time LO order parameter correlations given by 156

$$
\begin{aligned}
n\left(z, \mathbf{r}_{\perp}=0\right) & =\left\langle\Delta_{L O}^{*}(z) \Delta_{L O}(0)\right\rangle, \\
& \sim e^{-c_{1}(\ln z)^{6 / 5}} \cos \left(q_{0} z\right) .
\end{aligned}
$$

Although these $3 \mathrm{~d}$ anomalous effects are less dramatic and likely to be difficult to observe in practice, theoretically they are quite significant as they represent a qualitative breakdown of the mean-field and harmonic descriptions of the FFLO striped states.

\section{F. Phases and transitions}

\section{Topological defects}

Associated with its two compact Goldstone modes, $\phi_{ \pm}$ (equivalently $\phi(\mathbf{r})=\frac{1}{2}\left(\phi_{+}+\phi_{-}\right), u(\mathbf{r})=\frac{1}{2} a\left(\phi_{+}-\phi_{-}\right)$) the LO state admits two types of topological defects, characterized by integers $\vec{N}_{v}=\left(n_{+}, n_{-}\right)$defined by $\oint d \vec{\ell} \cdot \vec{\nabla} \phi_{ \pm}=2 \pi n_{ \pm}$. These equivalently correspond to superfluid vortices and edge dislocations in the striped PDW 167]. These are characterized by multiples of half-integers $n_{v}, n_{d}=\left(n_{+} \pm n_{-}\right) / 2$ and therefore allow four types of elementary defects: integer $(2 \pi, 0)$ vortex, integer dislocation $(0, a)$ and two half-integer vortexdislocation composites $(\pi, \pm a / 2)$. The latter composite fractional defects are allowed because a sign change in $\Delta_{L O}$ due to a $a / 2$-dislocation in $u$ is compensated by a $\pi$-vortex in $\phi$ (thereby preserving a single-valuedness of $\left.\Delta_{L O}\right)$ 37, 40, 66, 67]. In terms of the two coupled $\phi_{+}, \phi_{-}$ Goldstone modes, these correspond to an integer vortex in one and no vortex in the other superfluid phase.

Their thermodynamics and correlations can be treated via a mapping on a multi-component Coulomb gas

$$
\begin{aligned}
H_{C G}^{x y-s m}= & \frac{1}{2} \int_{\mathbf{q}}\left[\frac{\sqrt{\rho_{s}^{\perp} \rho_{s}^{\|}}}{\Gamma_{\mathbf{q}}^{x y}}\left|m_{\mathbf{q}, v}\right|^{2}+\frac{K q_{\perp}^{2}}{\Gamma_{\mathbf{q}}^{s m}}\left|m_{\mathbf{q}, d}\right|^{2}\right] \\
& +\sum_{\mathbf{r}_{i}}\left(E_{c}^{v} n_{\mathbf{r}_{i}, v}^{2}+E_{c}^{d} n_{\mathbf{r}_{i}, d}^{2}\right)
\end{aligned}
$$

where

$$
\begin{aligned}
\Gamma_{\mathbf{q}}^{x y} & =q_{\perp}^{2} \sqrt{\rho_{s}^{\perp} / \rho_{s}^{\|}}+q_{z}^{2} \sqrt{\rho_{s}^{\|} / \rho_{s}^{\perp}}, \\
\Gamma_{\mathbf{q}}^{s m} & =q_{z}^{2}+\lambda^{2} q_{\perp}^{4} .
\end{aligned}
$$

with $m_{\mathbf{q}, v}, m_{\mathbf{q}, d}$ the Fourier transforms of the vortex and dislocation densities. Equivalently, defects thermodynamics can be analyzed via duality transformation. In two dimensions it leads to a sine-Gordon-like model for dual $\tilde{\phi}, \tilde{\theta}$ fields characterizing fractional defects, with the dual Hamiltonian 37, 67, 171 .

$$
\tilde{H}_{S G}=\frac{1}{2} \int_{\mathbf{q}}\left[\frac{\Gamma_{\mathbf{q}}^{x y}}{\sqrt{\rho_{s}^{\perp} \rho_{s}^{\|}}}\left|\tilde{\phi}_{\mathbf{q}}\right|^{2}+\frac{\Gamma_{\mathbf{q}}^{s m} q_{0}^{2}}{K q_{\perp}^{2}}\left|\tilde{\theta}_{\mathbf{q}}\right|^{2}\right]-\int_{\mathbf{r}}\left[g_{\pi, a / 2} \cos (\pi \tilde{\phi}) \cos (\pi \tilde{\theta})+g_{2 \pi, 0} \cos (2 \pi \tilde{\phi})+g_{0, a} \cos (2 \pi \tilde{\theta})\right]
$$

It is convenient for analyzing the effects of defects on the LO state, particularly for a computation of their screening on long scales, unbinding, and for the analysis of the resulting disordered state. From the form (38) it is clear that (aside from an inconsequential anisotropy) the dual vortex sector described by $\tilde{\phi}$ has a standard sine-Gordon form. In contrast, the dual dislocation sector, described by $\tilde{\theta}$ is qualitatively modified by the highly nonlocal and qualitatively anisotropic smectic kernel, $\Gamma_{\mathbf{q}}^{s m}$.

A standard analysis gives the relative energetics of these defects, in the thermodynamic limit $\left(L_{\perp, z} \rightarrow \infty\right)$ given by

$$
E_{(0, a)}^{d} \sim K L \ll E_{(\pi, a / 2)}^{v-d} \sim \frac{\rho_{s}}{4} L \ln L+\frac{K}{4} L \ll E_{(2 \pi, 0)}^{v} \sim \rho_{s} L \ln L, \quad \text { for } L_{\perp, z} \sim L \rightarrow \infty
$$




$$
\begin{array}{ccccc}
F L_{S m} & \rightarrow & F L_{N} & \rightarrow & F L_{I} \\
\uparrow \mathrm{U}(1) & & \uparrow \mathrm{U}(1) & & \uparrow \mathrm{U}(1) \\
S F_{S m} & \rightarrow & S F_{N} & \rightarrow & S F_{I}
\end{array}
$$

TABLE I: Five phases that naturally emerge as disordered descendants of the LO (superfluid smectic, $S F_{S m}$ ) state.

Based on this energetics one may be tempted to conclude that in this limit (unless preempted by a first-order transition) it is the integer dislocation loop defects that proliferate first and the $\mathrm{LO}$ smectic preferentially disorders into a nematic superfluid, $S F_{N}$. However, in contrast to the $2 \mathrm{~d}$ KT mechanism [144, the $3 \mathrm{~d}$ disordering transitions take place when the relevant stiffness, renormalized by quantum and thermal fluctuations is continuously driven to zero at the transition, or takes place at a finite (rather than a vanishing) defects fugacity. For a thermal transition this roughly corresponds to a transition temperature set by the corresponding stiffnesses, $\bar{\rho}_{s}=\sqrt{\rho_{s}^{\|} \rho_{s}^{\perp}}$ and $K, B$. Thus, in principle by tuning these stiffnesses via imbalance and resonant interaction, a variety of phases can be accessed.

\section{Conventional phases and transitions}

By considering all possible basic combinations of spontaneously "broken" subset of spatial and gauge symmetries leads to an array of partially spatially-ordered paired superfluids and Fermi-liquid states, that are descendants of the smectic LO $\left(\mathrm{SF}_{S m}\right)$ state. These isotropic (I), nematic (N) and smectic (Sm) SF and FL states are summarized in Table I. It is notable that the isotropic superfluid, $S F_{I}$ exhibits a finite species imbalance and off-diagonal long-range order, symmetry-wise isomorphic to the polarized superfluid, $S F_{M}$ [79, 100, latter confined to the BEC side of the BCS-BEC crossover. In contrast, (as a descendant of the LO state expected to be stabilized by Fermi surfaces imbalance) the $S F_{I}$ state is realized in the BCS regime, something that has been searched for dating back to Sarma[117, but has not been possible within mean-field treatments, that instead predict an instability to phase separation [74, 79, 100. The isotropic Fermi liquid, $F L_{I}$ is isomorphic to the conventional normal state. Together these intermediate fluctuation-induced phases naturally interpolate between the fully gapped singlet (homogeneously and isotropic) BCS superconductor at zero imbalance and low temperature, and a polarized Fermi liquid at large imbalance and/or high temperature.

\begin{tabular}{|c|c|c|c|}
\hline phase/symmetry & $U(1)$ & $T_{\mathbf{q}_{0}}$ & $R$ \\
\hline$F L_{I}$ & $\sqrt{ }$ & $\sqrt{ }$ & $\sqrt{ }$ \\
\hline$F L_{N}$ & $\sqrt{ }$ & $\sqrt{ }$ & $\mathrm{X}$ \\
\hline$F L_{S m}$ & $\sqrt{ }$ & $\mathrm{X}$ & $\mathrm{X}$ \\
\hline$S F_{I}$ & $\mathrm{X}$ & $\sqrt{ }$ & $\sqrt{ }$ \\
\hline$S F_{N}$ & $\mathrm{X}$ & $\sqrt{ }$ & $\mathrm{X}$ \\
\hline$S F_{S m}$ & $\mathrm{X}$ & $\mathrm{X}$ & $\mathrm{X}$ \\
\hline
\end{tabular}

TABLE II: A summary of LO liquid crystal Fermi-liquid (FL) and superfluid (SF) phases, and corresponding order parameters and broken symmetries, indicated by X's. Unbroken symmetries (gauge $U(1)$, translational $T_{q_{0}}$, rotational $R$ ) are marked by check marks. The subscripts $I, N, S m$ respectively indicate the Isotropic, Nematic and Smectic orders.

\section{Topological phases via defects unbinding}

The phases discussed above can be complementarily characterized through unbinding of different combinations of topological defects. The smectic (whether SF LO state or FL smectic) to nematic transition is driven by unbinding of defects with edge dislocation charge, followed by transition into the isotropic state driven by proliferation of disclinations. The superfluid and Fermi-liquid version of these liquid crystal states are distinguished by unbinding defects with superfluid vortex charge.

However, a characterization in terms of topological defects also allows a distinction between topologically distinct phases with the same conventional order, where a Landau order parameter is insufficient to distinguish them. In fact because of the vanishing LO order parameter, description in terms of topological order is necessary even for the smectic LO state, distinguished from its more disordered descendants by the absence of unbound topological defects, in direct analogy with the quasi-longrange ordered state of the $2 \mathrm{~d}$ xy model.

A rich variety of possible phases and transitions is displayed in a schematic imbalance-detuning $P-1 / k_{F} a$ phase diagram, Fig 5. Increasing the imbalance suppresses the superfluid stiffness and drives the system toward a conventional Fermi liquid state, $F L_{I}$ at $h_{c 2}$. Conversely, a reduction in species imbalance primarily reduces the elastic moduli of the smectic pair-density wave by increasing its period $1 / q_{0}$ and thereby weakening the interaction between the LO domain-walls, driving the system toward a conventional isotropic and homogeneous superfluid $S F_{I}$ at $h_{c 1}$.

Thus, starting with the LO $S F_{S m}$ state and decreasing $h$ leads to the unbinding of the integer dislocations $(0, a)$, and a transition to an orientationally-ordered, i.e., a nematic "charge"-4 superfluid, $S F_{N}^{4}$. The later "charge"-4 feature of $S F_{N}^{4}$ naturally appears as the remaining secondary order parameter $\Delta_{s c}^{(4)}=\Delta_{L O}^{2}$ once the LO positional order $\Delta_{L O}$ is destroyed by unbinding of integer dislocation loops. Since in contrast, the "charge"-2 SF nematic order vanishes in the LO state, a direct transi- 
tion to it from the LO state can generically only proceed through a first-order transition.

Conversely, increasing $h$ starting with the LO $S F_{S m}$ is expected to lead to a suppression of $\rho_{s}$, a proliferation $(2 \pi, 0)$ vortices, and a transition to a $2 q$-smectic Fermi liquid, $F L_{S m}^{2 q}$, a non-superfluid periodic state with a wavevector that is twice the LO state. Alternatively, as suggested by the energetics in Eq. (39), if instead the lower-energy half-vortex dislocation defects $(\pi, a / 2)$ (or the $(\pi,-a / 2)$, but not both) unbinds first, a transition to a nematic Fermi liquid, $F L_{N}^{* *}$ (with the restored translational and $\mathrm{U}(1)$ charge symmetries) will take place. The resulting state is qualitatively distinct from the more conventional nematic (orientationally ordered) $F L_{N}$ phase in which both $(\pi, a / 2)$ and $(\pi,-a / 2)$ are proliferated. Both are also distinct from the nematically ordered $F L_{N}^{*}$ state, in which only integer dislocations, $(0, a)$ and integer vortices, $(2 \pi, 0)$ are unbound. One can envision a number of other states and phase transitions at low $h$ by further considering the disordering of the nematic superfluid, $S F_{N}^{4}$ by unbinding various patterns of disclinations and $\pi$-vortices. Many open questions remain about the relative energetics and detailed properties of these phases.

Above considerations lead to at least three topologically distinct Fermi liquid phases that naturally emerge from disordering of the LO $\left(S F_{S m}\right)$ phase by unbinding different combinations of allowed defects. Because the conventional vortex $(2 \pi, 0)$ and the conventional dislocation $(0, a)$ are composites of the fundamental defects $(\pi, \pm a / 2)$, the nonsuperfluid states $F L_{N}^{*}, F L_{N}^{* *}$ and their isotropic cousins $F L_{I}^{*}, F L_{I}^{* *}$ (in which disclinations are also unbound) are expected to be "fractionalized" [172, topologically distinct from their conventional Fermi liquid analogs, where $(\pi, \pm a / 2)$ are also unbound.

These novel phases are analogous to the putative phase-disordered fractionalized states obtained by unbinding double $(h c / e)$ vortices, studied extensively by Sachdev, and by Balents, Senthil, Fisher, and collaborators 172 174 in the context of high temperature superconductors. The resulting nonsuperfluid phase is distinguished from a conventional Fermi liquid by a gapped "vison", a $\mathbb{Z}_{2}$ defect that is a remnant of the fundamental $h c / 2 e$ vortex after the composite $h c / e$ (double) vortices proliferate.

The states $F L_{N}^{*}, F L_{N}^{* *}$ also bare a close relation to the collective mode fractionalization discussed by Sachdev, et al. 175 177. in the context of quantum paramagnetic phases, emerging from disordering a collinear spindensity wave. As with the $(U(1) \otimes U(1)) / \mathbb{Z}_{2}$ LO state, where the order parameter is a product of the superfluid and smectic order parameters, Eq. (16), there too the order parameter is of $\left(S_{2} \otimes U(1)\right) / \mathbb{Z}_{2}$ product form, encoding spatial modulation of the spin density, and therefore admits half-integer (vison-like) defects. Correspondingly, the phases and transitions can equivalently be captured via an effective Ising gauge theory. Its nonzero $\mathbb{Z}_{2}$ flux through a plaquette encodes the presence of a half- integer $(\pi, a / 2)$ defect. The Ising gauge field encodes the local $\mathbb{Z}_{2}$ redundancy of splitting the LO order parameter (16) into a "charge"-2 boson, $b_{\mathbf{r}}^{\dagger}=e^{-i \phi_{\mathbf{r}}}$, that creates a zero-momentum Cooper-pair (diatomic molecule) and a neutral boson, $\rho_{q, \mathbf{r}}^{\dagger}=e^{-i \theta_{\mathbf{r}}}$, that creates a density wave at the $\mathrm{LO}$ wavevector $q$. In the above notation this is the nonsuperfluid periodic state dubbed $F L_{S m}^{2 q}$, in which $(2 \pi, 0)$ vortices have proliferated, but dislocations remain bound. For a vison remaining gapped, the resulting nonsuperfluid nematic state is the topologically ordered $F L_{N}^{*}$, qualitatively distinct from a conventional $F L_{N}$ in which the vison is gapless. The two FL phases are separated by a deconfinement transition of vison condensation, $F L_{N^{-}}^{*} F L_{N}$, corresponding to a proliferation of the $(\pi, a / 2)$ fractional defects, that is expected to be in the inverted Ising universality class.

The variety of phases and transitions between them are summarized in the phase diagram, Fig 5 and a flow-chart, Fig.4. This rich fluctuations-driven phase behavior contrasts sharply with a direct LO-N transition (described by $U(1) \times U(1)$ Landau theory $H_{m f t}=r\left(\left|\Delta_{+}\right|^{2}+\left|\Delta_{-}\right|^{2}\right)+$ $\left.\lambda_{1}\left(\left|\Delta_{+}\right|^{4}+\left|\Delta_{-}\right|^{4}\right)+\lambda_{2}\left|\Delta_{+}\right|^{2}\left|\Delta_{-}\right|^{2}\right)$ found in mean-field theory.

\section{G. Fermionic excitations}

The subtlety of the LO and its descendent states is in addition to strongly fluctuating Goldstone modes, they exhibit gapless fermionic excitations that can be strongly coupled to the superfluid phase $\phi$ and smectic phonon $u$ Goldstone modes. A complete comprehensive treatment remains an open problem. However, a number of approximate numerical and analytical treatments has lead to a consistent picture 67, 134 137.

At large imbalance, near $h_{c 2}$ where $\Delta$ modulation is weak, the fermionic spectrum can be approximated by various $\mathbf{q}$ branches of the form Eq. (7) for the FF state. These lead to a novel paired superfluid with a Fermi surface of Bogoliubov quasi-particles.

Near $h_{c 1}$, a periodic form of $\Delta(\mathbf{r})$ is more appropriately treated as an array of domain-walls of width $\xi$ 134 137, rather than a single harmonic. Diagonalizing the BdG equations in their presence leads to a band of midgap states as in $1 \mathrm{~d}$ where this can be done exactly 67, 147, 178, corresponding to imbalanced fermionic atoms residing on the nodes of the solitons in $\Delta$ akin to the polyacetylene 180 . Consistent with the large imbalance regime this leads to a Fermi surface pockets [179] illustrated in Fig 9. Despite considerable progress a fully self-consistent quantum-mechanical treatment is still missing.

\section{H. Open questions}

While a broad range of phenomena associated with the Larkin-Ovchinnikov state has been explored, many inter- 


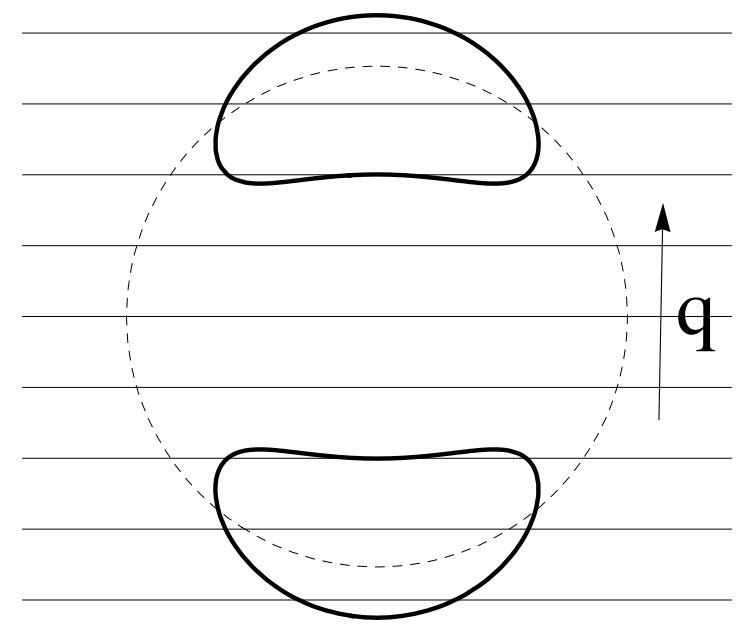

FIG. 9: An illustration of Fermi pockets (full curve) of the gapless Bogoliubov quasi-particles characteristic of the Larkin-Ovchinnikov ground state. The periodic array of domain-walls in $\Delta_{L O}(z)$, the associated wavevector $\mathbf{q}$, and the Fermi-surface of the underlying normal state (dashed circle) are also indicated.

esting and difficult questions remain open. Probably the most urgent of these is the long-standing question of the range of energetic stability of the crystalline superconductor. If the state is indeed stable over a sufficiently broad range of detuning and imbalance to be experimentally accessible, is its lowest energy form indeed the striped collinear LO type? While for large atomic clouds and shallow traps (such that LDA remains valid) only a small deformation of the LO state near the boundaries is expected, for tighter traps a more detailed treatment of the trap is necessary, and may lead to a distinct global form of the LO state, such as the "onion" and "radial" structures. To address such questions undoubtedly requires numerical solutions in experiment-specific geometries.

Furthermore, the nature of the $(2 \mathrm{~d}$ and $3 \mathrm{~d})$ transition into the LO state at the lower-critical Zeeman field $h_{c 1},[135$, and the extent to which it resembles a commensurate-incommensurate transition (as in 1d 134, 152]) remains an open question. More broadly, while a number of LO descendent states have been proposed their detailed phenomenology, stability to quantum and thermal fluctuations, as well the nature of the associated phase transitions remains wide open. Similarly to the LO state, these phases are expected to exhibit gapless fermionic excitations coupled to their Goldstone modes. Understanding the effects of these fermionic modes on the phases and the associated transitions remain an extremely interesting and challenging problem.

To summarize, I reviewed a wide range of fluctuation phenomena in a LO state, expected to be realizable in an imbalanced resonant Fermi gas. Combining a microscopic analysis with robust model-independent symmetry arguments predicts that the LO state in an isotropic trap is a gapless superfluid smectic liquid crystal. Consequently, the state is extremely sensitive to thermal fluctuations that destroy its long-range positional order even in three dimensions, replacing it by a quasi-long range order, characterized by power-law correlations akin to a system tuned to a critical point or two-dimensional xy-model systems. This exotic state also exhibits vortex fractionalization, where the basic superfluid vortex is half the strength of a vortex in a regular paired condensate, and is accompanied by half-dislocations in the LO smectic (layered) structure.

Analysis of the fluctuation-driven disordering of the LO smectic predicts a rich variety of descendant quantum liquid states, such as the superfluid $\left(S F_{N}\right)$ and Fermi liquid $\left(F L_{N}\right)$ nematics and the fractionalized nonsuperfluid states $\left(F L^{*}\right)$, that generically intervene between the LO state and the conventional BCS superfluid (at low population imbalance) and a conventional Fermi liquid (at high population imbalance). This phenomenology has a rich variety of experimental implications, many of which await detailed analysis.

\section{P-WAVE RESONANT BOSE GAS}

\section{A. Background}

A $p$-wave resonant Bose gas is another system that was recently predicted to exhibit quantum liquid crystal order. Its study was in part motivated by the interesting phenomenology found in $s$-wave resonant bosonic systems (e.g., in ${ }^{85} \mathrm{Rb}[26]$ ), that was demonstrated to exhibit a magnetic field-driven quantum Ising transition between molecular and atomic superfluids [27 29, [183, contrasting with a smooth BEC-BCS crossover in balanced fermionic isotopes.

Although instabilities intrinsic to resonant bosons 26, 159] challenge realization of such bosonic molecular condensates, many features of the phase diagram are expected to survive away from the resonance and/or reflected in the nonequilibrium phenomenology (before the onset of the instability) of a resonant Bose gas. Furthermore, recent extension of an $s$-wave resonant Bose gas to an optical lattice [184, 185] demonstrated the stabilization through a quantum Zeno mechanism proposed by Rempe [186, that dates back to Bethe's [187] analysis of the triplet linewidth in hydrogen.

The predictions [27, 29, 184, 185] for the $s$-wave case have been supported by recent density matrix renormalization group [188], exact diagonalization [189], and quantum Monte Carlo [190] studies. I am enthusiastic about similar progress in the substantially richer $p$-wave case, that I discuss below.

Experimental realization of two-species degenerate 
Bose gas with a $p$-wave Feshbach resonant inter-species interaction in ${ }^{85} \mathrm{Rb}-{ }^{87} \mathrm{Rb}$ mixtures 159 provides a direct motivation for the theoretical studies discussed below [53, 59]. Related studies of Bose condensation in $p$-(and higher) bands in optical lattices have also been carried out 61, 62, but are only tangentially relevant to the present focus of quantum liquid crystals.

\section{B. Summary}

The phenomenology of a balanced two-component $p$ wave resonant Bose gas is summarized by a temperaturedetuning phase diagram. As illustrated in Fig. 10 at

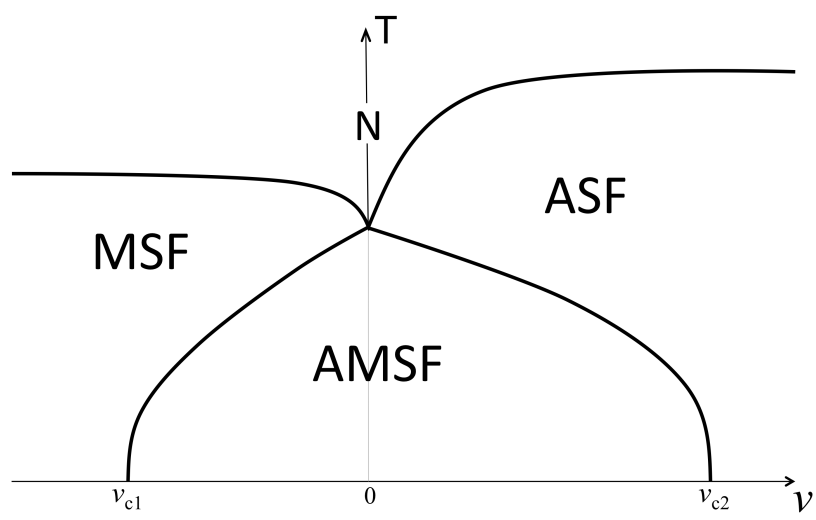

FIG. 10: Schematic temperature-detuning phase diagram for a balanced two-species $p$-wave resonant Bose gas. As illustrated, it exhibits atomic (ASF), molecular (MSF), and atomic-molecular (AMSF) superfluid phases. The novel AMSF state is characterized by a $p$-wave, molecular and a finite-momentum $Q$ (see Fig. 11) atomic superfluidity.

low temperature such two-component Bose gas generically exhibits three classes of superfluid phases, atomic (ASF), molecular (MSF) and atomic-molecular (AMSF) condensates.

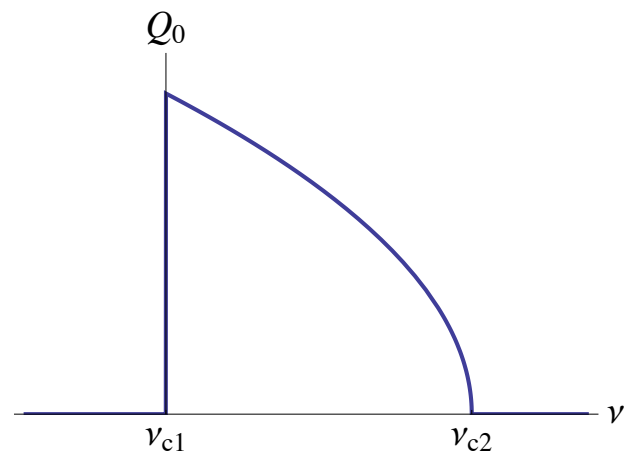

FIG. 11: The momentum $Q(\nu)$ characteristic of the AMSF (polar) state, ranging between zero and the $p$-wave FR widthdependent value.

The ASF appears at a large positive detuning (weak FR attraction) and low temperature, where one of the three combinations $\left(\mathrm{ASF}_{1}, \mathrm{ASF}_{2}, \mathrm{ASF}_{12}\right)$ of the ${ }^{85} \mathrm{Rb}$ and ${ }^{87} \mathrm{Rb}$ atoms are Bose-condensed into a conventional, uniform superfluid, and the $p$-wave ${ }^{85} \mathrm{Rb}-{ }^{87} \mathrm{Rb}$ molecules are energetically costly and therefore appear only as gapped excitations.

In the complementary regime of a large negative detuning, the attraction between two flavors of atoms is sufficiently strong so as to bind them into a tight $p$-wave hetero-molecules (e.g., ${ }^{85} \mathrm{Rb}-{ }^{87} \mathrm{Rb}$ molecule), which at low temperature condense into a $p$-wave molecular superfluid isomorphic to a spinor-1 condensate 60, 191 200. The latter is known to come in two forms, thereby predicting the $\ell_{z}=0$ "polar" $\left(\mathrm{MSF}_{\mathrm{p}}\right)$ and $\ell_{z}= \pm 1$ "ferromagnetic" $\left(\mathrm{MSF}_{\mathrm{fm}}\right)$ molecular $p$-wave superfluid phases, with their relative stability determined by the ratio $a_{0} / a_{2}$ of molecular spin-0 $\left(a_{0}\right)$ to molecular spin-2 $\left(a_{2}\right)$ scattering lengths.

Besides these fairly conventional uniform atomic and molecular BEC's, for intermediate detuning around a unitary point the gas is predicted to exhibits novel $\mathrm{AMSF}_{\mathrm{p}}$ and $\mathrm{AMSF}_{\mathrm{fm}}$ phases, characterized by a nonzero momentum $\hbar Q$ atomic condensate [53, 59, 61, that is a superposition of two atomic species. The momentum

$$
Q=\alpha m \sqrt{n_{m}} \sim \sqrt{\gamma_{p} \ell n_{m}} \lesssim \sqrt{\gamma_{p}} / \ell
$$

is tunable via the FR detuning, $\nu$, primarily entering through the molecular condensate $n_{m}(\nu) \lesssim 1 / \ell^{3}$ density, and sensitive to the FR width $\gamma_{p}$ 3. In addition to exhibiting an off-diagonal long-range order (ODLRO) of an ordinary superfluid the two $\mathrm{AMSF}_{\mathrm{p}, \mathrm{fm}}$ states (distinguished by the polar versus ferromagnetic nature of their $p$-wave molecular condensates) spontaneously partially break orientational and translational symmetries, akin to polar and smectic liquid crystals [48] and the putative Fulde-Ferrell-Larkin-Ovchinnikov states of imbalanced paired fermions 2, 5, 50, 51, 68, 69, 71, 79, 84, 100. discussed in the first part of this review. This state is a finite momentum, $\hbar Q$ spinor superfluid, akin to (but distinct from) a supersolid [125, 128.

The physical picture behind such a finite-momentum AMSF formation is quite clear and is illustrated as a cartoon in AMSF phase of Fig. 10. At intermediate detuning, where atomic gap closes within the MSF state, $p$-wave molecules decay via $\mathrm{FR}$ into a pair of atoms,

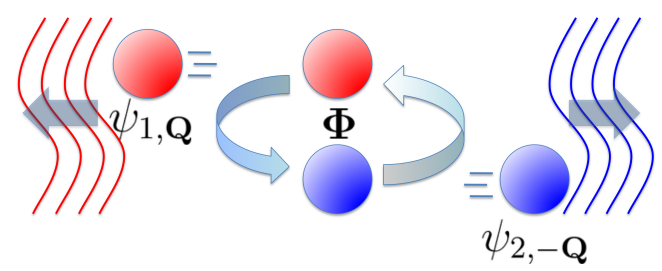

FIG. 12: A cartoon of a $p$-wave molecule decaying into two oppositely moving two species of atoms, illustrating a resonant mechanism for a finite momentum $\boldsymbol{Q}$ atomic superfluidity (indicated by wavy lines) in the AMSF phase. 
which (due to the $p$-wave nature of the molecules) are necessarily created at finite and opposite momenta, $\pm \mathbf{k}$, and therefore at low temperature form a finite momentum atomic condensate, AMSF. The energetic cost $\left(\sim Q^{2} / 2 m\right)$ of a finite momentum atomic condensation is balanced by the lowering of the energy $\left(\sim \alpha Q \sqrt{n_{m}}\right)$ through FR hybridization between closed-channel $p$-wave molecule and open-channel pair of atoms that is only possible at finite atomic momentum, giving $Q$ in Eq. (40).

As illustrated in Fig. 13, in the polar $\mathrm{AMSF}_{\mathrm{p}}$ state, $\boldsymbol{Q}$ aligns along the quantization axis along which the molecular condensate has a zero projection of its internal $\ell=1$ angular momentum. For the case of the ferromagnetic

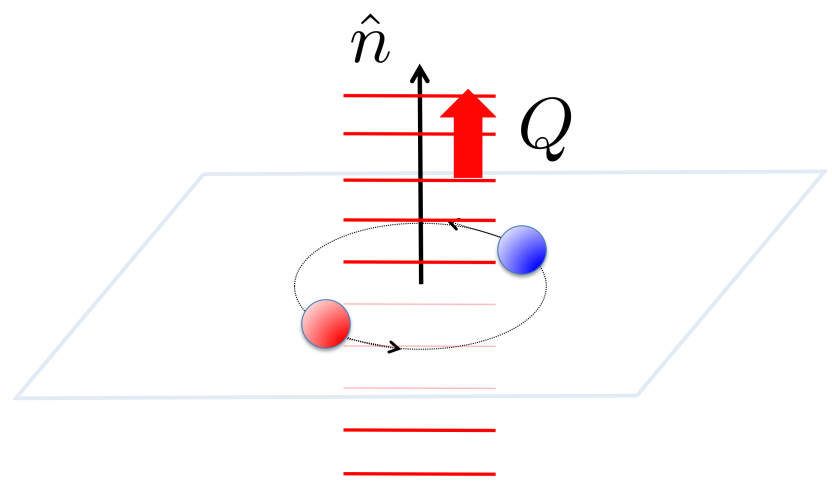

FIG. 13: Schematic of the $\mathrm{AMSF}_{\mathrm{p}}$ polar state. The thick arrow indicates the atomic condensate momentum $\boldsymbol{Q}$ and the $\hat{n}$ arrow denotes the quantization axis along which the projection of molecular internal orbital angular momentum vanishes.

$\mathrm{AMSF}_{\mathrm{fm}}$ state, $\boldsymbol{Q}$ lies in the otherwise isotropic plane, transverse to the $p$-wave molecular condensate axis, as illustrated in Fig. 14 .

As any neutral superfluid, ASF, MSF, and AMSF are each characterized by Bogoliubov modes, with long wavelength acoustic "sound" dispersions

$$
E_{\sigma}^{\mathrm{B}}(\mathbf{k}) \approx c_{\sigma} \hbar k,
$$

where $c_{\sigma}$ (with $\sigma=\mathrm{ASF}_{1,2,12}, \mathrm{MSF}_{\mathrm{p}, \mathrm{fm}}, \mathrm{AMSF}_{\mathrm{p}, \mathrm{fm}}$ ) are the associated sound speeds with standard Bogoliubov form $c_{\sigma} \approx \sqrt{g_{\sigma} n_{\sigma} / 2 m}$. In each of these SF states one Bogoliubov mode (and only one in the $\mathrm{ASF}_{i}$ states) corresponds to the overall condensate phase fluctuations. In addition, the $\mathrm{MSF}_{\mathrm{p}}$ exhibits two degenerate "transverse" Bogoliubov orientational acoustic modes. The $\mathrm{MSF}_{\mathrm{fm}}$ is also additionally characterized by one "ferromagnetic" spin-wave mode, $E_{k}^{\mathrm{MSF}}{ }_{\mathrm{fm}} \sim k^{2}$ and one gapped mode, consistent with the characteristics of a conventional spinor-1 condensate [194, 195].

Because $\mathrm{MSF}_{\mathrm{p}, \mathrm{fm}}$ are paired molecular superfluids, they also exhibit gapped single atom-like quasiparticles (akin to Bogoliubov excitations in a fermionic paired BCS state), that do not carry a definite atom number. These

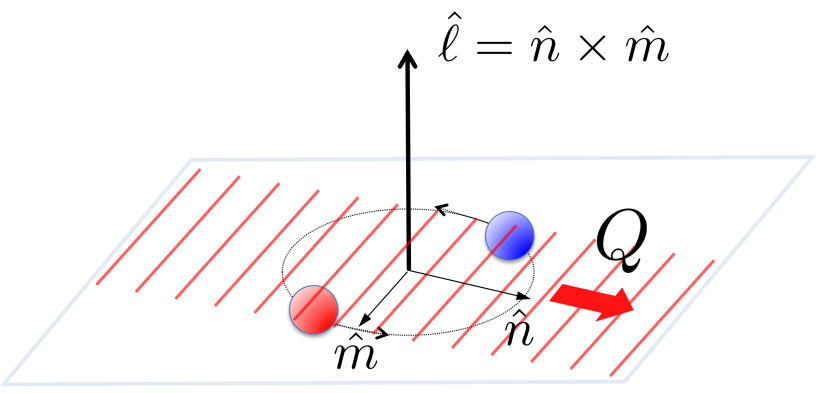

FIG. 14: Schematic of the $\mathrm{AMSF}_{\mathrm{fm}}$ ferromagnetic state. The thick arrow indicates the atomic condensate momentum $\boldsymbol{Q}$, lying in the plane transverse to the quantization axis $\hat{\ell}$, along which the projection of the molecular internal orbital angular momentum is $\ell_{z}=+1$.

single-particle excitations are "squeezed" by the presence of the molecular condensate, offering a mechanism to realize atomic squeezed states 201, that can be measured by interference experiments, similar to those reported in Ref. 202. The low-energy nature of these single-atom excitations is guaranteed by the vanishing of the gap at the MSF-AMSF transition at $\nu_{c}^{\mathrm{MSF}_{\mathrm{p}, \mathrm{fm}}-\mathrm{AMSF}_{\mathrm{p}, \mathrm{fm}}}$, with $E_{\mathrm{MSF}}^{\mathrm{gap}}\left(\nu_{c}\right)=0$.

In addition to conventional Bogoliubov modes the AMSF exhibits a Goldstone mode corresponding to the fluctuations of a relative phase between the two atomic condensate components. A spatially periodic collinear AMSF state exhibits a smectic-like anisotropic phonon mode, akin to striped FFLO states [51, 66, 67, 138, discussed in the first part of this review.

$$
\begin{aligned}
\omega_{\mathrm{AMSF}_{\mathrm{p}}}(\mathbf{k}) & =\sqrt{\left(B k_{z}^{2}+K k_{\perp}^{4}\right) / \chi_{-}}, \\
\omega_{\mathrm{AMSF}_{\mathrm{fm}}}(\mathbf{k}) & =\sqrt{\left(B k_{z}^{2}+k^{2}\left(K_{x} k_{x}^{2}+K_{y} k_{y}^{2}\right)\right) / \chi_{-}},
\end{aligned}
$$

and an orientational mode $\omega_{\mathrm{fm}}^{\gamma}$, associated with orientational symmetry breaking in $\mathrm{AMSF}_{\mathrm{fm}}$

$$
\begin{aligned}
& \omega_{+p}(\mathbf{k})=\sqrt{\frac{2 \rho_{s}}{\chi_{+} m} k}, \\
& \omega_{\mathrm{fm}}^{\gamma}(\mathbf{k})=\sqrt{\frac{J k^{2}\left[B k_{z}^{2}+k^{2}\left(K_{x} k_{x}^{2}+K_{y} k_{y}^{2}\right)\right]}{J \chi_{-} k^{2}+\kappa^{2} k_{y}^{2}}},
\end{aligned}
$$

where $B, K$ 's, $J, \kappa$, and $\chi$ are compressional and bending moduli characterizing the phases. 


\section{Microscopic model of a balanced $p$-wave resonant Bose gas}

As required by bosonic statistics a $p$-wave resonance takes place between two distinguishable bosonic atoms created by $\psi_{\sigma}^{\dagger}(\mathbf{r})=\left(\psi_{1}^{\dagger}(\mathbf{r}), \psi_{2}^{\dagger}(\mathbf{r})\right),\left(\right.$ e.g., ${ }^{85} \mathrm{Rb}$, ${ }^{87} \mathrm{Rb}$ ) [159, interacting through a closed-channel $\ell=$ 1 hetero-molecule created by a vector field operator $\phi^{\dagger}(\mathbf{r})=\left(\phi_{x}^{\dagger}, \phi_{y}^{\dagger}, \phi_{z}^{\dagger}\right)$. The corresponding Hamiltonian is given by,

$$
\begin{aligned}
\mathcal{H}= & \sum_{\sigma=1,2} \hat{\psi}_{\sigma}^{\dagger} \hat{\varepsilon}_{\sigma} \hat{\psi}_{\sigma}+\hat{\boldsymbol{\phi}}^{\dagger} \cdot \hat{\omega} \cdot \hat{\boldsymbol{\phi}}+\mathcal{H}_{b g} \\
& +\frac{\alpha}{2}\left(\hat{\boldsymbol{\phi}}^{\dagger} \cdot\left[\hat{\psi}_{1}(-i \boldsymbol{\nabla}) \hat{\psi}_{2}-\hat{\psi}_{2}(-i \boldsymbol{\nabla}) \hat{\psi}_{1}\right]+\text { h.c. }\right),
\end{aligned}
$$

where $\hat{\varepsilon}_{\sigma}=-\frac{1}{2 m} \nabla^{2}-\mu_{\sigma}, \hat{\omega}=-\frac{1}{4 m} \nabla^{2}-\mu_{m}$, with molecular chemical potential $\mu_{m}=\mu_{1}+\mu_{2}-\nu$, adjustable by a magnetic field dependent detuning $\nu$, latter the rest energy of the closed-channel molecule relative to a pair of open-channel atoms. A generalization to number and mass imbalanced mixtures (as studied for fermionic atoms [2, 5, 68, 69, 71, 79, 84, 100, remain to be explored [204].

For simplicity, above form is specialized to a rotationally invariant $\mathrm{FR}$ interaction, with $\hat{\omega}$ and $\alpha$ independent of the molecular component $i$. This is an approximation for the system of interest, ${ }^{85} \mathrm{Rb}^{-}{ }^{87} \mathrm{Rb}$ mixture, where indeed the $p$-wave FR around $B=257.8$ Gauss 159 is split into a doublet by approximately $\Delta B=0.6$ Gauss, similar to the fermionic case of ${ }^{40} \mathrm{~K}[3,12,14,203$. A more realistic, richer case remains to be explored.

The background (non-resonant) interaction density $\mathcal{H}_{b g}$ is the short-scale two-body interaction between spin and number densities characterized by atomic scattering lengths $a_{1}, a_{2}, a_{12}$ as well as molecular scattering lengths. A miscibility of a two-component atomic gas requires a condition on the corresponding atomic $s$-wave scattering lengths $a_{1} a_{2}>a_{12}^{2}$ [205, which may be problematic for the case of ${ }^{85} \mathrm{Rb}-{ }^{87} \mathrm{Rb}$ due to the negative background scattering length of ${ }^{85} \mathrm{Rb}$.

The corresponding imaginary time $(\tau)$ coherent state Lagrangian density is given by

$$
\begin{aligned}
\mathcal{L} & =\psi_{\sigma}^{*}\left(\partial_{\tau}-\frac{\nabla^{2}}{2 m}-\mu_{\sigma}\right) \psi_{\sigma}+\boldsymbol{\phi}^{*} \cdot\left(\partial_{\tau}-\frac{\nabla^{2}}{4 m}-\mu_{m}\right) \cdot \boldsymbol{\phi}+\frac{\lambda_{\sigma}}{2}\left|\psi_{\sigma}\right|^{4} \\
& +\lambda_{12}\left|\psi_{1}\right|^{2}\left|\psi_{2}\right|^{2}+g_{a m}\left(\left|\psi_{1}\right|^{2}+\left|\psi_{2}\right|^{2}\right)|\boldsymbol{\phi}|^{2}+\frac{g_{1}}{2}\left|\boldsymbol{\phi}^{*} \cdot \boldsymbol{\phi}\right|^{2}+\frac{g_{2}}{2}|\boldsymbol{\phi} \cdot \boldsymbol{\phi}|^{2} \\
& +\frac{\alpha}{2}\left(\boldsymbol{\phi}^{*} \cdot\left[\psi_{1}(-i \nabla) \psi_{2}-\psi_{2}(-i \nabla) \psi_{1}\right]+\text { c.c. }\right),
\end{aligned}
$$

where $\lambda_{i} \mathrm{~s}$ and $g_{i} \mathrm{~s}$ are the atomic and molecular $s$-wave pseudopotentials. Closely related models also arise in completely distinct physically contexts. These include quantum magnets that exhibit incommensurate spin liquids states [176] and bosonic atoms in the presence of spin-orbit interactions 54 .

The low-energy two-atom vacuum scattering in the above two-channel model can be computed exactly. It faithfully captures all the features of the low-energy $p$ wave resonant and $s$-wave nonresonant scattering phenomenology of the ${ }^{85} \mathrm{Rb}^{87} \mathrm{Rb} p$-wave Feshbach-resonant mixture [159]. By matching experiments in the dilute limit one can fix the model's key parameters [3, 59. The analysis at nonzero, balanced atomic densities leads to the predictions summarized above. All predictions are qualitatively robust, and can furthermore be made quantitatively accurate in a narrow-resonance limit.

\section{Phases, symmetries and Goldstone modes}

Qualitative features of the phase diagram for this system can be mapped out through a mean-field treatment of the Lagrangian, 45), supplemented by symmetry arguments and the analysis of fluctuations about the ordered state.

In the absence of periodic or disorder potential at sufficiently low temperatures a bosonic gas is always a superfluid, that in three dimensions exhibits Bose-Einstein condensation, characterized by scalar atomic, $\Psi_{\sigma}$ and/or 3 -vector molecular, $\mathbf{\Phi}$ complex order parameters. Thus, at low $\mathrm{T}$ the gas exhibits three classes of SF phases:

1. Atomic Superfluid (ASF), $\Psi_{\sigma} \neq 0$ and $\mathbf{\Phi}=0$

2. Molecular Superfluid (MSF), $\Psi_{\sigma}=0$ and $\boldsymbol{\Phi} \neq 0$

3. Atomic Molecular Superfluid (AMSF), $\Psi_{\sigma} \neq 0$ and $\boldsymbol{\Phi} \neq 0$

with the transition between them driven by the magnetic field-dependent detuning, $\nu$.

\section{Atomic superfluids: $A S F_{1}, A S F_{2}, A S F_{12}$}

For large positive detuning $\nu$, closed-channel molecules are gapped and the ground state is a molecular vac- 
uum, and a zero-momentum atomic condensate ASF. The latter itself comes in three forms: (i) $\mathrm{ASF}_{1}$ with $\Psi_{1} \neq 0, \Psi_{2}=0$, (ii) $\mathrm{ASF}_{2}$ with $\Psi_{1}=0, \Psi_{2} \neq 0$, (iii) $\mathrm{ASF}_{12}$ with $\Psi_{1} \neq 0, \Psi_{2} \neq 0$, separated by continuous phase transitions. For a balanced mixture $\tilde{\mu}_{1}=\tilde{\mu}_{2}$, the system exhibits a direct $\mathrm{N}-\mathrm{ASF}_{12}$ transition through a tetracritical point, $\tilde{\mu}_{1}=\tilde{\mu}_{2}=0$, that is believed to be in the decoupled universality class 206-208. All other transitions $\left(\mathrm{N}-\mathrm{ASF}_{1}, \mathrm{~N}-\mathrm{ASF}_{2}\right.$, and $\left.\mathrm{ASF}_{i}-\mathrm{ASF}_{12}\right)$ are in the XY universality class, breaking associated $U(1)$ symmetries. The phase boundaries and the values of the atomic condensate order parameters can be straightforwardly computed within mean-field theory [53, 59], but are modified by fluctuations 207, 208].

Within ASF phases, the spectrum of fluctuations can be straightforwardly computed by a Bogoliubov diagonalization of coupled atomic and molecular excitations, with details depending on which of the three possible $\mathrm{ASF}$ phases is studied. In general these states exhibit one Bogoliubov sound mode per broken atomic $U(1)$ symmetry, with one Goldstone mode in $\mathrm{ASF}_{1}$ and $\mathrm{ASF}_{2}$ phases and two in $\mathrm{ASF}_{12}$ [59.

\section{Molecular superfluids: $M S F_{p}$ and $M S F_{f m}$}

In the opposite limit of a large negative detuning, atoms are gapped, tightly bound into heteromolecules, that at low temperature condense into a $p$-wave molecular superfluid, MSF. In this regime of atomic vacuum, the gas reduces to that of interacting $p$-wave molecules, isomorphic to that of the extensively studied $F=1$ spinor condensate [60, 191 200, with the hyperfine spin $F$ here replaced by the orbital $\ell=1$ angular momentum of two constituent atoms.

Like $F=1$ spinor condensates, the $p$-wave molecular superfluid, MSF exhibits two distinct phases depending on the sign of the renormalized interaction coupling $g_{2}$ in Eq. 45 , or equivalently the sign of the difference $a_{0}^{(m)}-$ $a_{2}^{(m)}$ of the molecular $L=0$ and $L=2$ channels $s$-wave scattering lengths.

Polar molecular superfluid, $M S F_{p}$ :

For $g_{2}<0$ the ground state is the so-called "polar" molecular superfluid, $\mathrm{MSF}_{\mathrm{p}}$, characterized by a (collinear) order parameter $\boldsymbol{\Phi}=\Phi_{\mathrm{p}} e^{i \varphi} \hat{\boldsymbol{n}}$, with $\hat{\boldsymbol{n}}$ a real unit vector, $\varphi$ a (real) phase, and $\Phi_{\mathrm{p}}$ a (real) orderparameter amplitude, with the state corresponding to $\ell_{z}=0$ projection of the internal molecular orbital angular momentum along $\hat{\boldsymbol{n}}$. $\mathrm{MSF}_{\mathrm{p}}$ clearly spontaneously breaks rotational symmetry by its choice of the $\ell_{z}=0$ quantization axis $\hat{\boldsymbol{n}}$, and the global gauge symmetry, corresponding to a total atom number conservation. The low-energy order parameter manifold that characterizes $\mathrm{MSF}_{\mathrm{p}}$ is given by the coset space $\left(U(1) \otimes S_{2}\right) / \mathbb{Z}_{2}$, admitting half-integer "charge" vortices [200] akin to (but distinct from) the $s$-wave MSF [27-29].

The polar $\mathrm{MSF}_{\mathrm{p}}$ state exhibits three gapless Bogoliubov-like modes that are calculated in a standard way 59 . One corresponds to breaking of the global atom number conservation and two associated with breaking of rotational $O(3)$ symmetry [59, 194].

Ferromagnetic molecular superfluid, $M S F_{f m}$ :

Alternatively, for $g_{2}>0$ the ground state is the "ferromagnetic" molecular superfluid, $\mathrm{MSF}_{\mathrm{fm}}$, characterized by an (coplanar) order parameter $\boldsymbol{\Phi}=\frac{\Phi_{\mathrm{fm}}}{\sqrt{2}}(\hat{\boldsymbol{n}} \pm i \hat{\boldsymbol{m}})$, with $\hat{\boldsymbol{n}}, \hat{\boldsymbol{m}}, \hat{\boldsymbol{\ell}} \equiv \hat{\boldsymbol{n}} \times \hat{\boldsymbol{m}}$ a real orthonormal triad, $\Phi_{\mathrm{fm}}$ a real amplitude, and the state corresponds to $\ell_{z}= \pm 1$ projection of the internal molecular orbital angular momentum along the $\hat{\ell}$ axis. $\mathrm{MSF}_{\mathrm{fm}}$ spontaneously breaks the time reversal, the $O(3)$ rotational and the global gauge $U_{N}(1)$ symmetries, latter corresponding to a total atom number $N$ conservation. Inside $\mathrm{MSF}_{\mathrm{fm}}$ the low-energy order parameter manifold is that of the $O(3)=S U(2) / \mathbb{Z}_{2}$ group, corresponding to orientations of the orthonormal triad $\hat{\boldsymbol{n}}, \hat{\boldsymbol{m}}, \hat{\boldsymbol{\ell}}$.

As its hyperfine spinor-condensate cousin, the ferromagnetic $\mathrm{MSF}_{\mathrm{fm}}$ exhibits (only) two gapless Goldstone modes, one linear $(\propto k)$ conventional Bogoliubov mode associates with the broken global $U(1)$ gauge symmetry, and another quadratic $\left(\propto k^{2}\right)$ corresponding to the ferromagnetic order, with associated spin-waves [194] reflecting the precessional FM dynamics. This is despite the three-dimensionality of its $S O(3)$ coset space and can be traced back to the fact that the two components of the spinor are canonically conjugate and as a result combine into a single low-frequency $k^{2}$ mode.

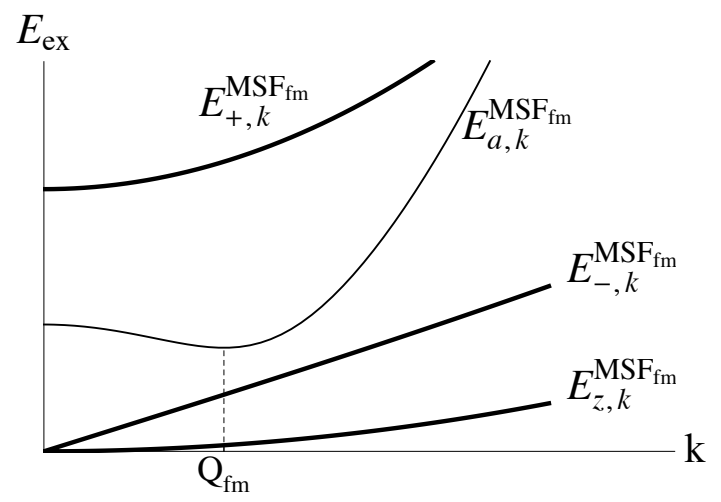

FIG. 15: Excitation spectrum for the ferromagnetic molecular superfluid, $\mathrm{MSF}_{\mathrm{fm}}$. The doubly-degenerate atomic spectrum (thin curves) exhibits a minimum gap at nonzero $k$, a precursor of finite momentum atomic condensation into the $\mathrm{AMSF}_{\mathrm{fm}}$. The molecular spectrum (thick curves), consists of a longitudinal gapless quadratic ferromagnetic spin-wave mode (lowest), a Bogoliubov sound mode and a quadratic gapped mode.

As illustrated in Fig. (15), while atomic excitations are gapped (confined into molecules), because of the $p$-wave FR coupling in 45 the minimum in the atomic spectrum occurs at a nonzero momentum, $Q_{p}$, a precursor of the Bose-condensation transition into a nonzero momentum AMSF state, when this atomic gap closes. 
3. Atomic-molecular superfluids: $A M S F_{p}$ and $A M S F_{f m}$

As detuning is further increased from a large negative value of the $\mathrm{MSF}_{\mathrm{p}, \mathrm{fm}}$ phases, for intermediate $\nu$ the gap to atomic excitations decreases, closing at a critical value of $\nu_{c}^{\mathrm{MSF}-\mathrm{AMSF}}$ and leading to atomic Bose-condensation into the corresponding $\mathrm{AMSF}_{\mathrm{p}}$ and $\mathrm{AMSF}_{f m}$ states. It is clear from the linear momentum dependence of the $p$ wave FR coupling in the Hamiltonian (44), that, quite generally, the MSF-AMSF transition is robustly into a nonzero-momentum atomic condensate, with $k=Q$, set by a balance of the $p$-wave FR hybridization and the atomic kinetic energies.

As with other (partially) crystalline states of matter [49 51, the nature of the resulting AMSF states depends on the symmetry of the crystalline order, encoded into the atomic condensate order parameter

$$
\Psi_{\sigma}(\mathbf{r})=\left(\begin{array}{c}
\Psi_{1}(\mathbf{r}) \\
\Psi_{2}(\mathbf{r})
\end{array}\right)=\sum_{Q_{n}}\left(\begin{array}{c}
\Psi_{1, Q_{n}} e^{i Q_{n} \cdot \mathbf{r}} \\
\Psi_{2,-Q_{n}} e^{-i Q_{n} \cdot \mathbf{r}}
\end{array}\right),
$$

via the associated set of the reciprocal lattice vectors, $\boldsymbol{Q}_{n}$ at which the condensation takes place. Determined by a detailed nature of interactions and fluctuations, typically the nature of crystalline order is challenging to deduce.

The nature and symmetries of these AMSF states furthermore qualitatively depends on the parent MSF, with the polar $\mathrm{AMSF}_{\mathrm{p}}$ and the ferromagnetic $\mathrm{AMSF}_{\mathrm{fm}}$ as two possibilities. In addition to the symmetries already broken in its MSF parent, by virtue of atomic condensation the AMSF state breaks the remaining $U_{\Delta N}(1)$ global gauge symmetry associated with the conservation of the difference in atom species number, $\Delta N$. Other symmetries that it breaks depend on the detailed structure of the $\mathrm{AMSF}_{\mathrm{fm}, \mathrm{p}}$ states.

Polar atomic-molecular superfluid, $A M S F_{p}$

The polar atomic-molecular superfluid $\mathrm{AMSF}_{\mathrm{p}}$ emerges from the polar molecular state, $\mathrm{MSF}_{\mathrm{p}}$. As illustrated in Fig 13 and is clear from the form of 45) in the $\mathrm{AMSF}_{\mathrm{p}}$ the finite momentum atomic condensate orders with $\boldsymbol{Q}$ locked along the molecular condensate field $\boldsymbol{\Phi}=\Phi_{\mathrm{p}} e^{i \varphi} \hat{\boldsymbol{n}}$. Hence, quite generically it is a Fulde-Ferrell like [50] collinear plane-wave state, that spontaneously breaks the time reversal symmetry, but not any additional spatial symmetries. A schematic phase diagram for this polar class of states is illustrated in Fig 16 The phase boundaries corresponding to the $\mathrm{MSF}_{\mathrm{p}}-\mathrm{AMSF}_{\mathrm{p}}$ and the $\mathrm{AMSF}_{\mathrm{p}}-\mathrm{ASF}$ transitions are given by

$$
\begin{aligned}
\nu_{\mathrm{c}}^{\mathrm{MSF}_{\mathrm{p}}-\mathrm{AMSF}_{\mathrm{p}}} & =-\left(g_{1}+g_{2}-2 \tilde{g}_{a m}\right) n_{m}, \\
& \approx-\frac{1}{2}\left(g_{1}+g_{2}-2 \tilde{g}_{a m}\right) n, \\
\nu_{\mathrm{c}}^{\mathrm{AMSF}_{\mathrm{p}}-\mathrm{ASF}} & =\left(2 \lambda-\tilde{g}_{a m}\right) n_{a}, \\
& \approx\left(2 \lambda-\tilde{g}_{a m}\right) n,
\end{aligned}
$$

The evolution of the order parameters across the phase diagram is illustrated in Fig 17 .

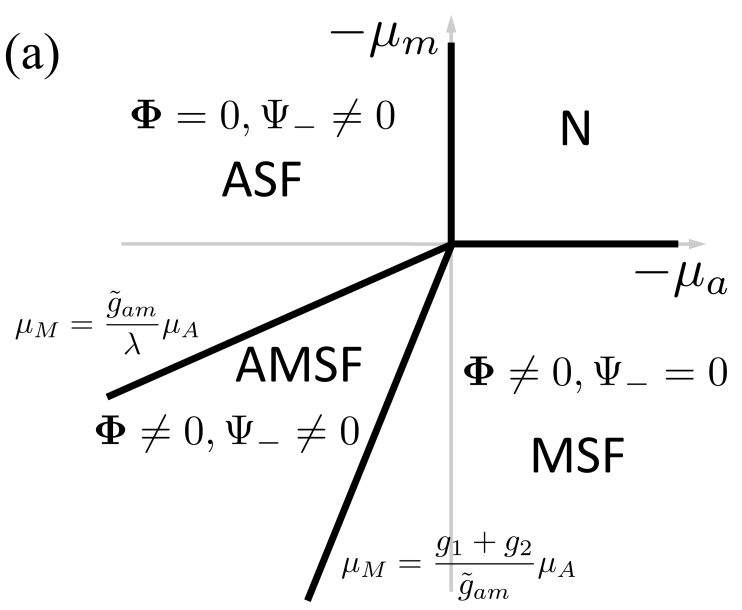

(b)

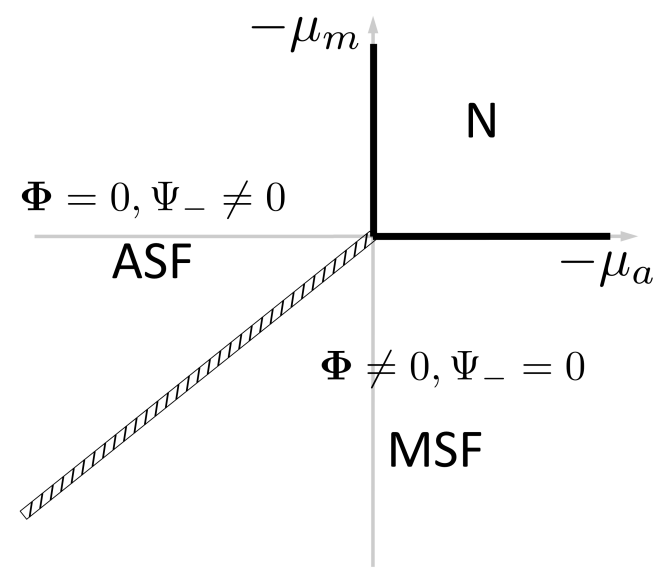

FIG. 16: Mean field phase diagrams for polar phase as a function of atomic and molecular chemical potentials, $\mu_{a}, \mu_{m}$, respectively. Ferromagnetic phase is similar but with different parameters. (a) For $\lambda\left(g_{1}+g_{2}\right)-\tilde{g}_{a m}^{2}>0$, all three superfluid phases, ASF, AMSF, and MSF appear and are separated by continuous phase transitions (thick black lines), (b) For $\lambda\left(g_{1}+g_{2}\right)-\tilde{g}_{a m}^{2}<0$, AMSF is unstable, and the ASF and MSF are separated by a first-order transition (hatched double line).

Despite a nonzero momentum $(Q)$ of its condensate, in its minimal form such $\mathrm{AMSF}_{p}$ state is a nematic (not smectic) superfluid that is anisotropic but spatially homogeneous. A detailed analysis 59] shows that Goldstone modes in this phase are governed by a Lagrangian density

$$
\begin{aligned}
\delta \mathcal{L}_{\mathrm{p}}= & \frac{1}{2} \chi_{+}\left(\partial_{\tau} \theta_{+}\right)^{2}+\frac{\rho_{s 0}}{m}\left(\nabla \theta_{+}\right)^{2} \\
& +\frac{1}{2} \chi_{-}\left(\partial_{\tau} \theta_{-}\right)^{2}+\frac{\rho_{0}}{m}\left(\partial_{z} \theta_{-}\right)^{2}+\frac{K}{2}\left(\nabla_{\perp}^{2} \theta_{-}\right)^{2},
\end{aligned}
$$

where the compressibilities $\chi_{ \pm}$and other couplings are functions of the microscopic parameters that have been computed in weakly interacting limit [59]. Thus, despite a translationally invariant nature of the $\mathrm{AMSF}_{p}$ phase, in addition to the conventional (linear in $k$ ) Bogoluibov 


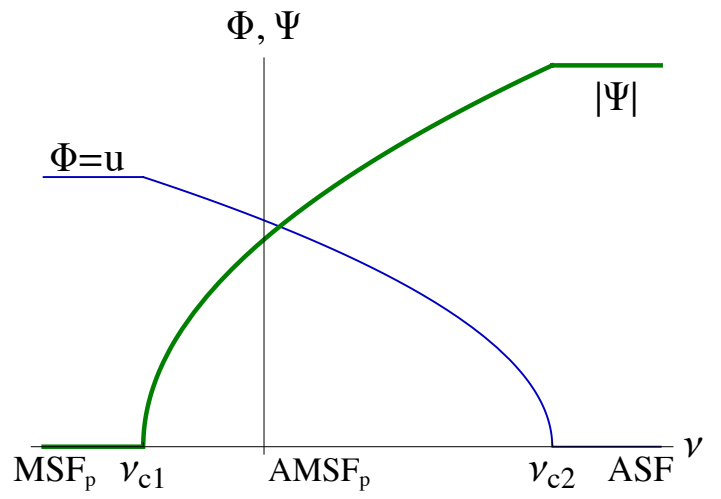

FIG. 17: Atomic (thick) and molecular (thin) order parameters versus the FR detuning $\nu$ for the polar phase, with $\nu_{\mathrm{c} 1}=\nu_{\mathrm{c}}^{\mathrm{MSF}_{\mathrm{p}}-\mathrm{AMSF}_{\mathrm{p}}}$ and $\nu_{\mathrm{c} 2}=\nu_{\mathrm{c}}^{\mathrm{AMSF}_{\mathrm{p}}-\mathrm{ASF}}$.

mode, $\theta_{+}$, akin to the FF state [50, 66, 67, 138] it exhibits a smectic-like Goldstone mode, $\theta_{-}$, with dispersion given in Eq. $42 \mathrm{a}$ [59].

Ferromagnetic atomic-molecular superfluid, $A M S F_{f m}$ Similarly, upon increase of the detuning, a finite- momentum atomic condensation from the $\mathrm{MSF}_{\mathrm{fm}}$ leads to the ferromagnetic atomic-molecular superfluid, $\mathrm{AMSF}_{\mathrm{fm}}$. In this state, a $p$-wave Feshbach resonant interaction leads to the energetic preference for a transverse orientation of the atomic condensate momentum $\boldsymbol{Q}$ to the molecular quantization axis, $\hat{\boldsymbol{\ell}}=\hat{\boldsymbol{n}} \times \hat{\boldsymbol{m}}$. Consequently, as illustrated in Fig 14 , the $\mathrm{AMSF}_{\text {fm }}$ state spontaneously breaks additional rotational symmetry of the uniaxial molecular state in the plane perpendicular to the molecular quantization axis $\hat{\ell}$. In fact the nature of spatial order inside the $\hat{\boldsymbol{n}}-\hat{\boldsymbol{m}}$ plane is not necessarily of co-linear striped order, and generically admits a $2 \mathrm{~d}$ crystalline condensate, nontrivially determined by interactions and fluctuations. Motivated by the polar version of AMSF, so far only a striped order has been investigated, with general form remaining an open problem. For such collinear order, a mean-field analysis predicts a FF (rather than LO [51]) type $\mathrm{AMSF}_{f m}$ state in the $\hat{\boldsymbol{n}} \boldsymbol{-} \hat{\boldsymbol{m}}$ plane. Thus, the $\mathrm{AMSF}_{\mathrm{fm}}$ state is a biaxial nematic superfluid, defined by $\boldsymbol{Q}$ and $\hat{\ell}$ axes.

The corresponding Goldstone modes Lagrangian density is given by:

$$
\begin{aligned}
\delta \mathcal{L}_{\mathrm{fm}} & \approx \frac{1}{2} \chi_{+}\left(\partial_{\tau} \theta_{+}\right)^{2}+\frac{\rho_{s 0}}{m}\left(\boldsymbol{\nabla} \theta_{+}\right)^{2}+\frac{1}{2} \chi_{-}\left(\partial_{\tau} \theta_{-}\right)^{2}+\frac{1}{2} B\left(\partial_{z} \theta_{-}\right)^{2}+\frac{1}{2} K_{x}\left(\boldsymbol{\nabla} \partial_{x} \theta_{-}\right)^{2}+\frac{1}{2} K_{y}\left(\boldsymbol{\nabla} \partial_{y} \theta_{-}\right)^{2} \\
& +i \kappa \partial_{y} \theta_{-} \partial_{\tau} \gamma+\frac{1}{2} J(\nabla \gamma)^{2}
\end{aligned}
$$

where the Goldstone mode $\gamma$ describes one additional fluctuating angle of the $\hat{\boldsymbol{m}}$ axis outside of the $\hat{\boldsymbol{n}} \boldsymbol{-} \hat{\boldsymbol{m}}$ plane, into the $\hat{\boldsymbol{\ell}}$ axis.

The biaxiality is expected and arises due to the vector $p$-wave order, characterized by a spinor $\boldsymbol{\Phi}_{f m}$, with the quantization axis, $\hat{\ell}$. The finite angular momentum, $\ell_{z}=$ \pm 1 along $\hat{\ell}$ distinguishes $\mathrm{AMSF}_{\mathrm{fm}}$ from $\mathrm{AMSF}_{\mathrm{p}}$ and leads to this additional Goldstone mode $\gamma$.

A straightforward diagonalization of the above Lagrangian leads to dispersions for three Goldstone modes inside the $\mathrm{AMSF}_{\mathrm{fm}}$ state:

$$
\begin{aligned}
& \omega_{f m}^{+}(\mathbf{k})=c_{+} k, \\
& \omega_{f m}^{-}(\mathbf{k})=\sqrt{\left[B k_{z}^{2}+k^{2}\left(K_{x} k_{x}^{2}+K_{y} k_{y}^{2}\right)\right] / \chi_{-}}, \\
& \omega_{f m}^{\gamma}(\mathbf{k})=\sqrt{\frac{J k^{2}\left[B k_{z}^{2}+k^{2}\left(K_{x} k_{x}^{2}+K_{y} k_{y}^{2}\right)\right]}{J \chi_{-} k^{2}+\kappa^{2} k_{y}^{2}}} .
\end{aligned}
$$

The anisotropic $\omega_{f m}^{\gamma}(\mathbf{k})$ dispersion corresponds to the ferromagnetic spin-waves in the plane of atomic condensate phase-fronts ("smectic layers") of the $p$-wave atomicmolecular condensate, $\mathrm{AMSF}_{\mathrm{fm}}$, reducing to the dispersion of $\mathrm{MSF}_{\mathrm{fm}}$ for a vanishing smectic order, with $B=0$.

\section{E. Phase transitions}

Phase transitions from the high temperature normal state into the ASF and MSF states have been wellexplored in the context of conventional and spinor superfluids.

The quantum MSF - AMSF phase transitions are more interesting and require a beyond mean-field treatment. In large part the complexity is associated with the nontrivial coupling of the Goldstone modes of the MSF phase to the finite-momentum order parameter condensing in the AMSF state.

Starting with the coherent-state microscopic Lagrangian, specializing to the $\mathrm{MSF}_{p}$ ordered state, and integrating out massive modes, one obtains the effective Lagrangian density

$$
\begin{aligned}
\mathcal{L}_{\mathrm{p}}= & \varepsilon_{+}^{-1}\left|\partial_{\tau} \psi_{-}\right|^{2}+\frac{1}{2 m}\left|\left(-i \boldsymbol{\nabla}-\alpha m \sqrt{\rho_{m 0}} \delta \hat{\boldsymbol{n}}\right) \psi_{-}\right|^{2} \\
& +\epsilon_{-}\left|\psi_{-}\right|^{2}+\frac{\lambda}{2}\left|\psi_{-}\right|^{4}+\frac{1}{2 g_{2}}\left(\partial_{\tau} \hat{n}\right)^{2}+\frac{\rho_{m 0}}{4 m}(\boldsymbol{\nabla} \hat{n})^{2} \\
& +\frac{1}{2 g}\left(\partial_{\tau} \varphi\right)^{2}+\frac{\rho_{m 0}}{4 m}(\nabla \varphi)^{2}
\end{aligned}
$$

that describes the quantum $\mathrm{MSF}_{p}-\mathrm{AMSF}_{p}$ transition. 
The critical field $\psi_{-}=\frac{1}{\sqrt{2}}\left(\psi_{1, Q}+\psi_{2,-Q}^{*}\right)$ is the finitemomentum atomic condensate order parameter, $\hat{\boldsymbol{n}}$ the $\ell_{z}=0$ quantization axis and $\phi$ the superfluid phase, defining the $\mathrm{MSF}_{p}$ state.

Thus, as can also be argued on symmetry grounds, the zero-temperature transition is indeed described by a quantum $((d+1)$-dimensional) de Gennes model (also known as the Ginzburg-Landau or Abelian-Higg's models) 48, akin to that for a normal-to-superconductor and nematic-to-smectic-A transitions. The role of the nematic director (gauge-field) is played by the $\ell_{z}=0$ quantization axis of the $p$-wave molecular condensate. Based on the extensive work for these systems [209, 210], in three (spatial) dimensions $(d=3)$ the effective gaugefield fluctuations drive this transition first-order.

Similarly, the quantum $\mathrm{MSF}_{\mathrm{fm}}-\mathrm{AMSF}_{\mathrm{fm}}$ transition is described by the effective low-energy Lagrangian density

$$
\begin{aligned}
\mathcal{L}_{\mathrm{fm}}= & \varepsilon_{+}^{-1}\left|\partial_{\tau} \psi_{-}\right|^{2}+\frac{1}{2 m}\left|\left(-i \nabla-\frac{\alpha m \sqrt{\rho_{m 0}}}{\sqrt{2}} \delta \hat{\boldsymbol{n}}\right) \psi_{-}\right|^{2} \\
& +\epsilon_{-}\left|\psi_{-}\right|^{2}+\frac{\lambda}{2}\left|\psi_{-}\right|^{4}+\frac{\rho_{m 0}}{8 m}(\boldsymbol{\nabla} \hat{\boldsymbol{n}})^{2}+\frac{\rho_{m 0}}{8 m}(\nabla \hat{\boldsymbol{m}})^{2} \\
& +\frac{1}{2 g}\left(\hat{\boldsymbol{n}} \cdot \partial_{\tau} \hat{\boldsymbol{m}}\right)^{2}
\end{aligned}
$$

It is distinguished from the polar case by the additional biaxial order whose fluctuations are characterized by $\hat{\boldsymbol{m}}$.

\section{F. Topological defects}

In addition the smooth low-energy configurations of these Goldstone modes, the compact nature of the order parameters characterizing the ASF, MSF and AMSF states admits a rich variety of topological defects. These singular excitations are crucial to a complete characterization of the states and their disordering, particularly in the case of non-meanfield (e.g., partially disordered) states that are not uniquely characterized by a Landau order parameter. In addition to the conventional vortices in the $\mathrm{ASF}_{i}$ states and well explored defects in the spin-1 $\mathrm{AMSF}_{p, f m}$ condensates, the $\mathrm{AMSF}_{p, f m}$ exhibit interesting combination of vortex, dislocation and domain-wall defects similar to those discussed in the context of FFLO and other striped superfluids. While some preliminary studies have been carried out [59], their detailed analysis, response to rotation, and realization remain subjects of future research.

\section{SUMMARY AND CONCLUSION}

In this review I discussed two recently studied quantum liquid crystal orders, proposed to be realizable in $s$-wave resonant fermionic and $p$-wave resonant bosonic atomic gases. The key ingredient is the interactiondriven superfluid condensation at a nonzero momentum, that in an isotropic trap spontaneously partially breaks orientational and in some cases translational symmetry. The main state that generically appears at low temperature is a quantum superfluid smectic. A rich variety of other phases results from partially disordering this state by unbinding various combinations of topological defects associated with compact nature of the broken symmetries. An interesting interplay ensues between Goldstone modes, associated spontaneously broken gauge and spatial symmetries and the fermionic and bosonic singleparticle excitations. Although a general formulation of such states is credibly established many interesting open questions remain.

\section{ACKNOWLEDGMENTS}

I thank A. Vishwanath and Sungsoo Choi for collaborations on which much of this review is based [53, [59, 66]. I acknowledge fruitful discussions with V. Gurarie, M. Hermele, D. Huse, and M. Levin. This work was supported by the National Science Foundation through grants DMR-1001240 and MRSEC DMR-0820579.
[1] I. Bloch, J. Dalibard, W. Zwerger, Rev. Mod. Phys. 80, 885 (2008).

[2] W. Ketterle and M. Zwierlein, "Making, probing and understanding ultracold Fermi gases", in Ultracold Fermi Gases, Proceedings of the International School of Physics "Enrico Fermi", Course CLXIV, Varenna, 20 - 30 June 2006, edited by M. Inguscio, W. Ketterle, and C. Salomon.

[3] V. Gurarie and L. Radzihovsky, Ann. of Phys. 322, 2 (2007).

[4] S. Giorgini, L.P. Pitaevskii, and S. Stringari, Rev. Mod. Phys. 80, 1215 (2008).

[5] L. Radzihovsky, D E. Sheehy, Rep. Prog. Phys. 73, 076501 (2010)
[6] C.A. Regal, M. Greiner, and D.S. Jin, Phys. Rev. Lett. 92, 040403 (2004).

[7] M.W. Zwierlein, C.A. Stan, C.H. Schunck, S.M.F. Raupach, A.J. Kerman, and W. Ketterle, Phys. Rev. Lett. 92, 120403 (2004).

[8] J. Kinast, S.L. Hemmer, M.E. Gehm, A. Turlapov, and J.E. Thomas, Phys. Rev. Lett. 92, 150402 (2004).

[9] M. Bartenstein, A. Altmeyer, S. Riedl, S. Jochim, C. Chin, J.H. Denschlag, and R. Grimm, Phys. Rev. Lett. 92, 120401 (2004).

[10] T. Bourdel, L. Khaykovich, J. Cubizolles, J. Zhang, F. Chevy, M. Teichmann, L. Tarruell, S.J.J.M.F. Kokkelmans, and C. Salomon, Phys. Rev. Lett. 93, 050401 (2004). 
[11] J. Zhang, E. G. M. van Kempen, T. Bourdel, L. Khaykovich, J. Cubizolles, F. Chevy, M. Teichmann, L. Tarruell, S. J. J. M. F. Kokkelmans, and C. Salomon, Phys. Rev. A 70, 030702(R) (2004).

[12] J. P. Gaebler, J. T. Stewart, J. L. Bohn, D. S. Jin, Phys. Rev. Lett. 98, 200403 (2007).

[13] S. S. Botelho and C. A. R. S. de Melo, J. Low Temp. Phys. 140, 409 (2005).

[14] V. Gurarie, L. Radzihovsky and A.V. Andreev, Phys. Rev. Lett. 94, 230403 (2005).

[15] C.-H. Cheng and S.-K. Yip, Phys. Rev. Lett. 95, 070404 (2005).

[16] D.M. Eagles, Phys. Rev. 186, 456 (1969).

[17] A.J. Leggett, in Modern Trends in the Theory of Condensed Matter, edited by A. Pekalski and R. Przystawa, (Springer-Verlag, Berlin, 1980).

[18] P. Nozières and S. Schmitt-Rink, J. Low Temp. Phys. 59, 195 (1985).

[19] C.A.R. Sá de Melo, M. Randeria, and J.R. Engelbrecht, Phys. Rev. Lett. 71, 3202 (1993).

[20] E. Timmermans, K. Furuya, P.W. Milonni, and A.K. Kerman, Physics Letters A 285, 228 (2001).

[21] M. Holland, S.J.J.M.F. Kokkelmans, M.L. Chiofalo, and R. Walser, Phys. Rev. Lett. 87, 120406 (2001).

[22] Y. Ohashi and A. Griffin, Phys. Rev. Lett. 89, 130402 (2002);

[23] A.V. Andreev, V. Gurarie, and L. Radzihovsky, Phys. Rev. Lett. 93, 130402 (2004).

[24] J. Stajic, J.N. Milstein, Q. Chen, M.L. Chiofalo, M.J. Holland, and K. Levin, Phys. Rev. A 69, 063610 (2004).

[25] M. L. Olsen, J. D. Perreault, T. D. Cumby, D. S. Jin Phys. Rev. A 80, 030701(R) (2009).

[26] S. L. Cornish, N. R. Claussen, J. L. Roberts, E. A. Cornell, and C. E. Wieman, Phys. Rev. Lett. 85, 1795 (2000).

[27] L. Radzihovsky, J. Park, and P. Weichman, Phys. Rev. Lett. 92, 160402 (2004).

[28] M. W. J. Romans, R. A. Duine, S. Sachdev, and H. T. C. Stoof, Phys. Rev. Lett. 93, 020405 (2004).

[29] L. Radzihovsky, P. Weichman, and J. Park, Annals of Physics 323, 2376 (2008).

[30] see e.g., Refs 1 5 and references therein.

[31] R. A. Barankov, L. S. Levitov, B. Z. Spivak, Phys. Rev. Lett. 93, 160401 (2004).

[32] E. Altman and A. Vishwanath, Phys. Rev. Lett. 95, 110404 (2005).

[33] S. A. Kivelson, E. Fradkin, V. J. Emery, Nature 393, 550 (1998).

[34] E. Fradkin, S. A. Kivelson, Phys. Rev. B 59, 8065 (1999).

[35] V. Oganesyan, S. Kivelson, E. Fradkin, Phys. Rev. B 64, 195109 (2001).

[36] E. Berg, E. Fradkin, S. A. Kivelson, Phys. Rev. B 79, 064515 (2009).

[37] E. Berg, E. Fradkin, S. A. Kivelson, Nature Phys. 5, 830 (2009).

[38] S. Sachdev, Annals Phys. 303226 (2003).

[39] D. F. Agterberg and H. Tsunetsugu, Nature Physics 4 639 (2008).

[40] D. F. Agterberg, S. Mukherjee, and Z. Zheng, Phys. Rev. Lett. 100, 017001 (2008).

[41] P. Bak, M.H. Jensen, J. Phys. C (Solid State) 13 L88 (1980).

[42] D. Belitz, T. R. Kirkpatrick, and A. Rosch, Phys. Rev.
B 73, 054431 (2006).

[43] A. M. Berridge, A. G. Green, S. A. Grigera, B. D. Simons Phys. Rev. Lett. 102, 136404 (2009).

[44] A. A. Koulakov, M. M. Fogler, and B. I. Schklovskii, Phys. Rev. Lett. 76, 499 (1996); Phys. Rev. B 54, 1853 (1996).

[45] M. P. Lilly, K. B. Cooper, J. P. Eisenstein, L. N. Pfeiffer, and K. W. West, Phys. Rev. Lett. 82, 394 (1999).

[46] A. H. MacDonald and M. P. A. Fisher, Phys. Rev. B 61, 5724 (2000).

[47] L. Radzihovsky, A. T. Dorsey, Phys. Rev. Lett. 88, 216802 (2002).

[48] P. deGennes and J. Prost, The Physics of Liquid Crystals, Clarendon Press, Oxford 1993.

[49] P. Chaikin and T.C. Lubensky, Principles of Condensed Matter Physics, Cambridge University Press, Cambridge 1995.

[50] P. Fulde and R.A. Ferrell, Phys. Rev. 135, A550 (1964).

[51] A.I. Larkin and Yu.N. Ovchinnikov, Zh. Eksp. Teor. Fiz. 47, 1136 (1964) [Sov. Phys. JETP 20, 762 (1965)].

[52] A. Sedrakian, J. Mur-Petit, A. Polls, and H. Müther, Phys. Rev. A 72, 013613 (2005).

[53] L. Radzihovsky and S. Choi, Phys. Rev. Lett. 103, 095302 (2009).

[54] C.-M. Jian, H. Zhai, Phys. Rev B 84, 060508 (2011).

[55] S. K. Yip, Phys. Rev. A 83, 043616 (2011).

[56] Z.-Q. Yu and H. Zhai, Phys. Rev. Lett. 107, 195305 (2011).

[57] C. J. Wang, G. Chao, C. M. Jian, and H. Zhai, Phys. Rev. Lett. 105, 160403 (2010).

[58] Y. J. Lin, K. Jimenez-Garcia, and I. B. Spielman, Nature 471, 83 (2011).

[59] S. Choi and L. Radzihovsky, Phys. Rev. A 84, 043612 (2011).

[60] M. Vengalattore, S. R. Leslie, J. Guzman, D. M. Stamper-Kurn, Phys. Rev. Lett. 100, 170403 (2008).

[61] A. B. Kuklov, Phys. Rev. Lett. 97, 110405 (2006).

[62] W. V. Liu and C. Wu, Phys. Rev. A 74, 013607 (2006).

[63] Y. L. Loh, N. Trivedi, arXiv:0907.0679

[64] Z. Cai, Y. Wang, C. Wu, Phys. Rev. A 83, 063621 (2011).

[65] Z. Zhang, X. Li, W. V. Liu, arXiv:1105.3387

[66] L. Radzihovsky and A. Vishwanath, Phys. Rev. Lett. 103, 010404, (2009).

[67] L. Radzihovsky, Phys. Rev. A 84, 023611 (2011).

[68] M.W. Zwierlein, A. Schirotzek, C.H. Schunck, and W. Ketterle, Science 311, 492 (2006).

[69] G.B. Partridge, W. Li, R.I. Kamar, Y. Liao, and R.G. Hulet, Science 311, 503 (2006).

[70] Y. Shin, M.W. Zwierlein, C.H. Schunck, A. Schirotzek, and W. Ketterle, Phys. Rev. Lett. 97, 030401 (2006).

[71] S. Nascimb'ene, N. Navon, K.J. Jiang, L. Tarruell, M. Teichmann, J. McKeever, F. Chevy, and C. Salomon, Phys. Rev. Lett. 103, 170402 (2009).

[72] R. Combescot, Europhys. Lett. 55, 150 (2001).

[73] W.V. Liu and F. Wilczek, Phys. Rev. Lett. 90, 047002 (2003).

[74] P.F. Bedaque, H. Caldas, and G. Rupak, Phys. Rev. Lett. 91, 247002 (2003).

[75] H. Caldas, Phys. Rev. A 69, 063602 (2004).

[76] J. Carlson and S. Reddy, Phys. Rev. Lett. 95, 060401 (2005).

[77] T.D. Cohen, Phys. Rev. Lett. 95, 120403 (2005).

[78] P. Castorina, M. Grasso, M. Oertel, M. Urban and D. 
Zappalà, Phys. Rev. A 72, 025601 (2005).

[79] D.E. Sheehy and L. Radzihovsky, Phys. Rev. Lett. 96, 060401 (2006).

[80] C.-H. Pao, S.-T. Wu, and S.-K. Yip, Phys. Rev. B 73, 132506 (2006).

[81] D.T. Son and M.A. Stephanov, Phys. Rev. A 74, 013614 (2006).

[82] A. Bulgac, M. Forbes, and A. Schwenk, Phys. Rev. Lett 97, 020402 (2006).

[83] J. Dukelsky, G. Ortiz, and S.M.A. Rombouts, and K. van Houcke, Phys. Rev. Lett. 96, 180404 (2006).

[84] T. Mizushima, K. Machida, and M. Ichioka, Phys. Rev. Lett. 94, 060404 (2005).

[85] K. Yang, Phys. Rev. Lett. 95, 218903 (2005).

[86] K. Yang and S. Sachdev, Phys. Rev. Lett. 96, 187001 (2006).

[87] P. Pieri and G.C. Strinati, Phys. Rev. Lett. 96, 150404 (2006).

[88] J. Kinnunen, L. M. Jensen, and P. Törmä, Phys. Rev. Lett. 96, 110403 (2006).

[89] W. Yi and L.-M. Duan, Phys. Rev. A 73, 031604 (2006).

[90] F. Chevy, Phys. Rev. Lett. 96, 130401 (2006).

[91] L. He, M. Jin, and P. Zhuang, Phys. Rev. B 73, 214527 (2006).

[92] T.N. De Silva and E.J. Mueller, Phys. Rev. A 73, 051602 (2006).

[93] M. Haque and H.T.C. Stoof, Phys. Rev. A 74, 011602 (2006).

[94] S. Sachdev and K. Yang, Phys. Rev. B 73, 174504 (2006).

[95] X.-J. Liu and H. Hu, Europhys. Lett. 75, 364 (2006).

[96] C. Chien, Q. Chen, Y. He, and K. Levin, Phys. Rev. Lett. 97, 090402 (2006).

[97] K.B. Gubbels, M.W.J. Romans, and H.T.C. Stoof, Phys. Rev. Lett. 97, 210402 (2006).

[98] W. Yi and L.-M. Duan, Phys. Rev. A 74, 013610 (2006).

[99] C.-H. Pao and S.-K. Yip, J. Phys. Cond. Mat. 18, 5567 (2006).

[100] D.E. Sheehy and L. Radzihovsky, Ann. of Phys. 322, 1790 (2007).

[101] D.E. Sheehy and L. Radzihovsky, Phys. Rev. B 75, 136501 (2007).

[102] J.-P. Martikainen, Phys. Rev. A 74, 013602 (2006).

[103] M. M. Parish, F.M. Marchetti, A. Lamacraft, and B.D. Simons, Nature Phys. 3, 124 (2007).

[104] A. Bulgac, M. Forbes, Phys. Rev. Lett. 101, 215301, 2008.

[105] M. M. Parish, S. K. Baur, E. J. Mueller, D. A. Huse, Phys. Rev. Lett. 99, 250403 (2007).

[106] D. E. Sheehy, Phys. Rev. A 79, 033606 (2009).

[107] Y. Nishida and D. T. Son, Phys. Rev. Lett. 97, 050403 (2006);

[108] R. Haussmann, W. Rantner, S. Cerrito, and W. Zwerger, Phys. Rev. A 75, 023610 (2007).

[109] P. Nikolić and S. Sachdev, Phys. Rev. A 75, 033608 (2007).

[110] M. Y. Veillette, D. E. Sheehy and L. Radzihovsky, Phys. Rev. A 75, 043614 (2007).

[111] M. Veillette, E. G. Moon, A. Lamacraft, L. Radzihovsky, S. Sachdev, D.E. Sheehy, Phys. Rev. A 78, 033614 (2008).

[112] N. Prokof'ev, B. Svistunov, Phys. Rev. B 77, 020408(R) (2008).

[113] S. Pilati, S. Giorgini, Phys. Rev. Lett. 100030401
(2008).

[114] K. Van Houcke, F. Werner, E. Kozik, N. Prokofev, B. Svistunov, M. Ku, A. Sommer, L. W. Cheuk, A. Schirotzek, M. W. Zwierlein, arXiv:1110.3747

[115] B. S. Chandrasekhar, Appl. Phys. Lett. 1, 7 (1962).

[116] A.M. Clogston, Phys. Rev. Lett. 9, 266 (1962).

[117] G. Sarma, J. Phys. Chem. Solids 24, 1029 (1963).

[118] M. Alford, J.A. Bowers, and K. Rajagopal, Phys. Rev. D 63, 074016 (2001).

[119] J.A. Bowers and K. Rajagopal, Phys. Rev. D 66, 065002 (2002).

[120] R. Casalbuoni and G. Nardulli, Rev. Mod. Phys. 76, 263 (2004).

[121] R. Combescot and C. Mora, Europhys. Lett. 68, 79 (2004).

[122] Y.-P. Shim, R.A. Duine, and A.H. MacDonald, Phys. Rev. A 74, 053602 (2006).

[123] Recent experiments found evidence of a FFLO state in heavy fermion, $\mathrm{CeCoIn}_{5}$ and in organic $\kappa$-(BEDT$\mathrm{TTF})_{2} \mathrm{Cu}(\mathrm{NCS})_{2}$ superconductors. See e.g., A. Bianchi, R. Movshovich, C. Capan, P.G. Pagliuso, and J.L. Sarrao, Phys. Rev. Lett. 91, 187004 (2003); H.A. Radovan, N.A. Fortune, T.P. Murphy, S.T. Hannahs, E.C. Palm, S.W. Tozer, and D. Hall, Nature 425, 51 (2003), and R. Lortz, Y. Wang, A. Demuer, P. H. M. Böttger, B. Bergk, G. Zwicknagl, Y. Nakazawa, and J. Wosnitza, Phys. Rev. Lett. 99, 187002 (2007).

[124] Y. Liao, A. S C. Rittner, T. Paprotta, W. Li, G. B. Partridge, R. G. Hulet, S. K. Baur, E. J. Mueller, Nature 467, 567 (2010).

[125] A.F. Andreev and I.M. Lifshitz, Zh. Eksp. Teor. Fiz. 56, 2057 (1969) [Sov. Phys. JETP 29, 1107 (1969)].

[126] G.V. Chester, Phys. Rev. A 2, 256 (1970).

[127] A.J. Leggett, Phys. Rev. Lett. 25, 1543 (1970).

[128] E. Kim and M.H.W. Chan, Nature 427, 225 (2004); Science 305, 1941 (2004).

[129] A LO state is qualitatively distinguished from a supersolid by the absence of a zero momentum condensate. While a conventional supersolid also breaks translational and gauge symmetry, it is characterized by two independent order parameters, in contrast to a LO state's single product order parameters. This puts LO state into a distinct univesality class, that is more appropriately referred to as a "pair-density wave" (PDW) 130.

[130] H.-D. Chen, O. Vafek, A. Yazdani, S.-C. Zhang, Phys. Rev. Lett. 93, 187002 (2004).

[131] The stability of the FFLO is significantly extended in optical lattices 63, 64 and in one dimension 105. However, our focus here is on a lattice-free realization, where rich liquid-crystal phenomenology is realized.

[132] S.-T. Wu, C.-H. Pao, S.-K. Yip, Phys. Rev. B 74, 224504 (2006).

[133] Y. He, C.-C. Chien, Q. Chen, K. Levin Phys. Rev. A 75, 021602(R) (2007).

[134] K. Machida and H. Nakanishi, Phys. Rev. B 30, 122 (1984).

[135] H. Burkhardt and D. Rainer, Ann. Physik 3, 181-194 (1994).

[136] S. Matsuo, S. Higashitani, Y. Nagato, and K. Nagai, J. Phys. Soc. Japan 67, 280 (1998).

[137] N. Yoshida, and S.-K. Yip, Phys. Rev. A 75, 063601 (2007).

[138] H. Shimahara, J. Phys. Soc. Jpn. 67, 1872 (1998).

[139] L. D. Landau, in Collected Papers of L. D. Landau, 
edited by D. ter Haar (Gordon and Breach, New York, 1965), p. 209; L. D. Landau and E. M. Lifshitz, Statistical Physics (Pergamon, London, 1969), p. 403.

[140] R. E. Peierls, Helv. Phys. Acta Suppl. 7, 81 (1934).

[141] N.D. Mermin, H. Wagner, Phys. Rev. Lett. 17, 1133 (1966).

[142] P.C. Hohenberg, Phys. Rev. 158, 383 (1967).

[143] V.L. Berezinskii, Zh. Eksp. Teor. Fiz. 59, 907 (1970) [Sov. Phys. JETP 32, 493 (1971)]; Zh. Eksp. Teor. Fiz. 61, 1144 (1971) [Sov. Phys. JETP 34, 610 (1972)].

[144] J.M. Kosterlitz and D.J. Thouless, J. Phys. C 6, 1181 (1973).

[145] J. Toner and D. R. Nelson, Phys. Rev. B 23, 316 (1981).

[146] J.R. Schrieffer, Theory of Superconductivity, Perseus, 1999.

[147] K. Samokhin, Phys. Rev. B 81, 224507 (2010).

[148] C.A. Regal and D.S. Jin, Phys. Rev. Lett. 90, 230404 (2003).

[149] The FFLO-N transition is only continuous within meanfield theory, and can be argued to be either generically driven first-order by fluctuations. 139, 151 or split into intermediate phases as illustrated in Fig 5 . This observation is quite generic and is due to the fact that $\mathrm{LO}$ state spontaneously breaks both translational and rotational symmetries, while the conventional paired SF breaks neither. Consequently, at least two continuous transitions are required.

[150] For a review see e.g.: V.L. Pokrovsky, A. L. Talapov and P. Bak in Solitons, edited by S. E. Trullinger, V. E. Zakharov and V. L. Pokrovsky (North Holland, Amsterdam, 1986), Chap. 3, pp. 71127.

[151] S. A. Brazovskii, Zh. Eksp. Teor. Fiz. 68, 175 (1975) [Sov. Phys. JETP 41, 85 (1975)].

[152] K. Yang, Phys. Rev. B 63, 140511 (2001).

[153] E. Zhao, W. V. Liu Phys. Rev. A 78, 063605 (2008).

[154] P.-G. de Gennes, Superconductivity of Metals and Alloys, Benjamin, New York, 1966.

[155] M. Tinkham, Introduction to Superconductivity, McGraw-Hill, 1996.

[156] G. Grinstein and R. A. Pelcovits, Phys. Rev. Lett. 47, 856 (1981).

[157] D. M. Stamper-Kurn, A. P. Chikkatur, A. Gorlitz, S. Inouye, S. Gupta, D. E. Pritchard, and W. Ketterle, Phys. Rev. Lett. 83, 2876 (1999).

[158] J. Steinhauer, R. Ozeri, N. Katz, and N. Davidson, Phys. Rev. Lett. 88, 120407 (2002)

[159] S. B Papp, J. M Pino, R. J Wild, S Ronen, C. E Wieman, D. S Jin, E. A Cornell, Phys. Rev. Lett. 101, 135301 (2008).

[160] Additional Berry phase effects may arise and qualitatively modify the dynamics, as argued in e.g., Ref 161.

[161] S. Fujimoto, arXiv:1008.5183

[162] A. Caille', C. R. Acad. Sci. Ser. B 274, 891 (1972).

[163] J. Als-Nielsen, J. D. Litster, R. J. Brigeneau, M. Kaplan, C. R. Safinya, A. Lindegaard-Andersen, and S. Mathiesen, Phys. Rev. B 22, 312 (1980).

[164] Z. Hadzibabic, P. Krüger, M. Cheneau, B. Battelier, and J. Dalibard, Nature 441, 1118 (2006).

[165] C.-L. Hung, X. Zhang, N. Gemelke, and C. Chin, Nature 470, 236 (2011).

[166] L. Golubovic, Z. Wang, Phys. Rev. Lett. 692535 (1992).

[167] These integer vector defects, $\vec{N}_{v}$ are associated with the fundamental group $\Pi_{1}$ of the torus $U(1) \otimes U(1), 168$ that characterizes the low-energy manifold of Goldstone modes of the LO state. In this respect the LO superfluid has similarities to other $U(1) \otimes U(1)$ systems, such as easy-plane spinor-1 condensates 169 and two-gap superconductors, e.g., $\mathrm{MgB}_{2}$ 170.

[168] In terms of the "rotated" Goldstone modes $\phi=\frac{1}{2}\left(\phi_{+}+\right.$ $\left.\phi_{-}\right)$and $\theta=\frac{1}{2}\left(\phi_{+}-\phi_{-}\right)$, the low-energy LO manifold is described as a half-twisted torus, $(U(1) \otimes U(1)) / \mathbb{Z}_{2}$. Because of the structure of the Hamiltonian the energetics is best analyzed in terms of $\phi, \theta$, while topological constraints on defects are most transparently implemented in terms $\phi_{ \pm}$.

[169] D. Podolsky, S. Chandrasekharan, A. Vishwanath, Phys. Rev. B 80, 214513 (2009).

[170] E. Babaev, Phys. Rev. Lett. 89, 067001 (2002).

[171] C. Lin, X. Li, and W. Vincent Liu, Phys. Rev. B 83, 092501 (2011).

[172] T. Senthil and M. P. A. Fisher, Phys. Rev. B. 62, 7850 (2000); ibid, 63, 134521 (2001).

[173] S. Sachdev, Phys. Rev. B 45, 389 (1992).

[174] L. Balents, M. P. A. Fisher, and C. Nayak, Phys. Rev. B 60, 1654 (1999).

[175] S. Sachdev, Quantum Phase Transitions, Cambridge University Press, Cambridge, 1999.

[176] S. Sachdev, Annals Phys. 303, 226 (2003).

[177] Z. Nussinov, J. Zaanen, J. Phys. IV France 12, 245 (2002); Physica Status Solidi B 236, 332 (2003).

[178] Quantum Theory of Many-Body Systems Techniques and Applications, A. M. Zagoskin, New York, Springer, 1998.

[179] In contrast, in 1d the effective chemical potential generically lies in the gap between Andreev bands 147. Thus, a 1d LO state exhibits only collective low-energy excitations $\phi, \theta$, consistent with its Luttinger-liquid description 152 , 153 .

[180] W. P. Su, J. P. Schrieffer, A. J. Heeger, Phys. Rev. Lett. 42, 1698 (1979); Phys. Rev. B 22, 2099 (1980).

[181] S. Mukerjee, C. Xu, and J. E. Moore, Phys. Rev. Lett. 97, 120406 (2006).

[182] We distinguish two independent $U(1)$ symmetries, $U_{N}(1)$ and $U_{\Delta N}(1)$, associated with independent total atom number $N=N_{1}+N_{2}+2 N_{m}$ conservation and the difference $\Delta N=N_{1}-N_{2}$ conservation, respectively.

[183] Y. W. Lee and Y. L. Lee, Phys. Rev. B. 70, 224506 (2004).

[184] S. Diehl, M. Baranov, A. J. Daley, and P. Zoller, Phys. Rev. B 82, 064509 (2010).

[185] S. Diehl, M. Baranov, A. J. Daley, and P. Zoller, Phys. Rev. B 82, 064510 (2010).

[186] N. Syassen, D. M. Bauer, M. Lettner, T. Volz, D. Dietze, J. J. Garcia-Ripoll, J. I. Cirac, G. Rempe, and S. Dürr, Science 320, 1329 (2008).

[187] H. A. Bethe Handbuch der Physik (1933), second edition, Vol. 24/1, pp. 452-462.

[188] S. Ejima, M. J. Bhaseen, M. Hohenadler, F. H. L. Essler, H. Fehske, and B. D. Simons, Phys. Rev. Lett. 106, 015303 (2011).

[189] M. J. Bhaseen, A. O. Silver, M. Hohenadler, and B. D. Simons, Phys. Rev. Lett. 103, 265302 (2009).

[190] L. Bonnes and S. Wessel, Phys. Rev. Lett. 106, 185302 (2011).

[191] M. R. Matthews, D. S. Hall, D. S. Jin, J. R. Ensher, C. E. Wieman, E. A. Cornell, F. Dalfovo, C. Minniti, and 
S. Stringari, Phys. Rev. Lett. 81, 243 (1998).

[192] J. Stenger, S. Inouye, D. M. Stamper-Kurn, H.-J. Miesner, A. P. Chikkatur, and W. Ketterle, Nature 396, 345 (1998).

[193] D. M. Stamper-Kurn, M. R. Andrews, A. P. Chikkatur, S. Inouye, H.-J Miesner, J. Stenger, and W. Ketterle, Phys. Rev. Lett. 80, 2027 (1998).

[194] T.-L. Ho, Phys. Rev. Lett. 81, 742 (1998).

[195] T. Ohmi and K. Machida, J. Phys. Soc. Japan 67, 1822 (1998).

[196] T.-L. Ho and S. K. Yip, Phys. Rev. Lett. 84, 4031 (2000).

[197] F. Zhou, Phys. Rev. Lett. 87, 080401 (2001).

[198] E. Demler and F. Zhou, Phys. Rev. Lett. 88, 163001 (2002).

[199] R. Barnett, A. Turner, and E. Demler, Phys. Rev. Lett. 97, 180412 (2006)

[200] S. Mukerjee, C. Xu, and J. E. Moore, Phys. Rev. Lett. 97, 120406 (2006).

[201] H. J. Kimble, Quantum fluctuations in quantum optics - squeezing and related phenomena, les Houches, Session
LIII, 1990 (Elsvier, 1992) and references therein.

[202] C. Orzel, A. K. Tuchman, M. L. Fenselau, M. Yasuda, and M. A. Kasevich, Science 291, 2386 (2001).

[203] C. A. Regal, C. Ticknor, J. L. Bohn, and D. S. Jin, Phys. Rev. Lett. 90, 053201 (2003).

[204] For unequal masses FR interaction is given by $H_{F R}=$ $\alpha \hat{\boldsymbol{\phi}}^{\dagger} \cdot\left[\frac{m_{1}}{m_{1}+m_{2}} \hat{\psi}_{1}(-i \boldsymbol{\nabla}) \hat{\psi}_{2}-\frac{m_{2}}{m_{1}+m_{2}} \hat{\psi}_{2}(-i \boldsymbol{\nabla}) \hat{\psi}_{1}\right]+$ h.c. as required to preserve Galilean invariance.

[205] B. D. Esry, C. H. Greene, J. P. Burke, Jr., and J. L. Bohn, et al., Phys. Rev. Lett. 78, 3594 (1997).

[206] K.-S. Liu and M. E. Fisher, J. Low Temp. Phys. 10, 655 (1973).

[207] J.M. Kosterlitz, D.R. Nelson, and M.E. Fisher, Phys. Rev. B 13, 412 (1976).

[208] P. Calabrese, A. Pelissetto, and E. Vicari, Phys. Rev. B 67, 054505 (2003)

[209] B. I. Halperin, T. C. Lubensky, and S. K. Ma, Phys. Rev. Lett. 32, 292 (1974).

[210] S. Coleman and E. Weinberg, Phys. Rev. D 7, 1888 (1973). 